\title{
A Tiny Eruptive Filament as a Flux-Rope Progenitor and Driver of a Large-Scale CME and Wave
}

\author{
V.V. Grechnev ${ }^{1}$ • A.M. Uralov ${ }^{1}$. \\ A.A. Kochanov ${ }^{1}$. I.V. Kuzmenko ${ }^{2}$. \\ D.V. Prosovetsky ${ }^{1}$. Ya.I. Egorov ${ }^{1}$. \\ V.G. Fainshtein ${ }^{1}$ L.K. Kashapova ${ }^{1}$
}

Received ; accepted

(C) Springer $\bullet \bullet \bullet \bullet$

\begin{abstract}
A solar eruptive event SOL2010-06-13 observed with the Atmospheric Imaging Assembly (AIA) of the Solar Dynamics Observatory (SDO) has been extensively discussed in the contexts of the CME development and an associated extremeultraviolet (EUV) wave-like transient in terms of a shock driven by the apparent CME rim. Continuing the analysis of this event, we have revealed an erupting flux rope, studied its properties, and detected wave signatures inside the developing CME. These findings have allowed us to establish new features in the genesis of the CME and associated EUV wave and to reconcile all of the episodes into a single causally-related sequence. (1) A hot $11 \mathrm{MK}$ flux rope developed from the structures initially associated with a compact filament system. The flux rope expanded with an acceleration of up to $3 \mathrm{~km} \mathrm{~s}^{-2}$ one minute before a hard X-ray burst and earlier than any other structures, reached a velocity of $420 \mathrm{~km} \mathrm{~s}^{-1}$, and then decelerated to about $50 \mathrm{~km} \mathrm{~s}^{-1}$. (2) The CME development was driven by the expanding flux rope. Closed coronal structures above the rope got sequentially involved in the expansion from below upwards, came closer together, and apparently disappeared to reveal their common envelope, the visible rim, which became the outer boundary of the cavity. The rim was probably associated with the separatrix surface of a magnetic domain, which contained the pre-eruptive filament. (3) The rim formation was associated with a successive compression of the upper active-region structures into the CME frontal structure (FS). When the rim was formed, it resembled a piston. (4) The disturbance responsible for the consecutive CME formation episodes was excited by the flux rope inside the rim, and then propagated outward. EUV structures arranged at different heights started to accelerate, when their trajectories in the distance-time diagram were
\end{abstract}

\footnotetext{
${ }^{1}$ Institute of Solar-Terrestrial Physics SB RAS, Lermontov St. 126A, Irkutsk 664033, Russia email: grechnev@iszf.irk.ru email: uralov@iszf.irk.ru

${ }^{2}$ Ussuriysk Astrophysical Observatory, Solnechnaya St. 21, Primorsky Krai, Gornotaezhnoe 692533, Russia email: kuzmenko_irina@mail.ru
} 
crossed by that of the fast front of this disturbance. (5) Outside the rim and FS, the disturbance propagated like a blast wave, manifesting in a type II radio burst and a leading part of the EUV transient. Its main, trailing part was the FS, which consisted of swept-up 2 MK coronal loops enveloping the expanding rim. The wave decelerated and decayed into a weak disturbance soon afterwards, being not driven by the trailing piston, which slowed down.

Keywords: Filament Eruptions; Coronal Mass Ejections; Shock Waves; Type II Bursts

\section{Introduction}

A solar eruptive event in active region (AR) 11079 at S21 W82 was observed on 13 June 2010 from about 05:30 to 05:50 (all times are referred to UT) with the Atmospheric Imaging Assembly (AIA) on board the Solar Dynamics Observatory (SDO; Lemen et al., 2012). Observations with the Sun Earth Connection Coronal and Heliospheric Investigation instrument suite (SECCHI; Howard et al., 2008) on the Solar-Terrestrial Relations Observatory (STEREO; Kaiser et al., 2008) from a different vantage point complement the picture of the event. This event has been extensively discussed in the contexts of the CME development (Patsourakos, Vourlidas, and Stenborg, 2010) and an associated extreme-ultraviolet (EUV) wave-like transient in terms of a piston shock driven by the apparent rim of the CME bubble (Ma et al., 2011, Kozarev et al., 2011, Gopalswamy et al., 2012, Eselevich and Eselevich, 2012, Kouloumvakos et al. 2014). Nevertheless, some important questions remain unanswered. It is still unclear where the flux rope was located, how it evolved, and which properties it had. It is uncertain how the CME was formed and what were the progenitors of its structural components.

Patsourakos, Vourlidas, and Stenborg (2010) followed the lift-off of the CME bubble in the SOL2010-06-13 event. The authors detected an eruptive filament, whose rise caused the rise of surrounding loops which eventually formed an EUV cavity. They concluded that the bubble was formed from a set of pre-existing loops during the main flare phase, while the rise and possible instability of the filament was considered as the possible CME trigger. The upper limit for the size of a hypothetical pre-existing flux rope was estimated to be very small, about $20 \mathrm{Mm}$.

Eselevich and Eselevich (2013) measured the expansion of the rising coronal loops starting from $\approx 30 \mathrm{Mm}$ and found their sequential involvement in the motion from below upwards during the CME formation. Having not noticed the eruptive filament, the authors proposed that the source of the CME was a magnetic tube emerging with a high speed from below the photosphere.

In spite of high-resolution multi-wavelength observations by SDO/AIA and Extreme UltraViolet Imager (EUVI) on STEREO-A and many efforts applied by several researchers, the flux rope escapes detection so far. The origin and regime of the CME-related wave remain conjectural. Based on a sophisticated thermodynamic magnetohydrodynamic (MHD) model, Downs et al. (2012) simulated a detailed evolution of the EUV wave in this event in realistic coronal 
conditions. The authors concluded that its outer, propagating component had properties of a fast-mode wave, but their analysis could not ascertain the wave excitation scenario. It is still unclear where and how the presumable shock wave developed.

Genesis of the flux rope, CME formation, and shock wave excitation scenario are common long-standing issues for many similar events. Addressing these issues promises reconciliation of existing concepts with observational challenges and progress in understanding eruptive events and underlying processes.

The basic guidelines to solve these problems are provided by the standard flare model ('CSHKP'; Carmichael, 1964, Sturrock, 1966, Hirayama, 1974; , Kopp and Pneuman, 1976) and its later developments. According to Hirayama (1974), the flare current sheet forms due to the lift-off of a filament, whose eruption is driven by an MHD instability of an increasing current in the filament. This can be the torus instability governed by the Lorentz force (Anzer, 1978, Chen, 1989, 1996). A twisted flux rope can be formed from an initial sheared configuration like a filament (van Ballegooijen and Martens, 1989, Uralov, 1990; Inhester, Birn, and Hesse, 1992. Longcope and Beveridge, 2007). Observational studies confirm the formation of flux ropes during flares (see, e.g., Asai et al., 2004; Qiu et al., 2007, Miklenic, Veronig, and Vršnak 2009) and their concurrent impulsive acceleration (Zhang et al., 2001, Temmer et al., 2008, 2010). The accelerating flux rope must produce an MHD disturbance. Propagating into surrounding regions, where the fast-mode speed is lower, the disturbance must rapidly steepen into a shock (Grechnev et al. 2011b, 2014b: Afanasyev, Uralov, and Grechnev, 2013), and then expand ahead of the CME like a decelerating blast wave for some time. If the CME is slow, then the shock eventually decays. Otherwise, the frontal part of the shock changes to the bow-shock regime.

Different events, ranging from the GOES B-class up to the X-class, exhibited these scenarios. The active role of filaments or similar structures as progenitors of flux ropes (Uralov et al., 2002, Grechnev et al., 2006a) was confirmed by observations (Chen, Bastian, and Gary, 2014, Grechnev et al., 2014a, 2015). The excitation by such structures of waves, which rapidly steepened into the shocks manifesting in 'EUV waves' and type II bursts, was first demonstrated by Grechnev et al. (2011b) and then confirmed in later studies of different events (see, e.g., Grechnev et al., 2013a, 2014b, 2015).

The 13 June 2010 event allows us to confront the outlined picture, which we develop, with different views of the authors, who studied this event previously, in order to verify our scenarios, specify and elaborate the conjectures widely invoked. Our new in-depth analysis of this eruptive event pursues the major unanswered questions of the genesis of the flux rope and its properties; how was the CME formed; where and how was the wave excited. We have revealed the developing flux rope and the appearance of an impulsively excited wave inside the forming CME, and studied some of their properties.

The fact that the development of neither the flux rope nor the shock wave have been detected previously indicates that various temperature ranges should be examined using sensitive image processing. Section 2 addresses these issues and our measurement techniques. Using them, we then analyze the observations and discuss the results. Section 3 considers the geometry of the CME bubble and 
the orientation of the flux rope. Section 4 is devoted to the flux rope. Section 5 analyzes the CME formation. Section 6 addresses the wave signatures. Section 7 discusses the origin of the observed structures, compares the findings with a traditional view, and presents an updated scenario of an eruptive event inferred from the observations. Section 8 summarizes the outcome from our analysis, outlines its implications, and finishes with concluding remarks.

\section{Methodical Issues}

\subsection{SDO/AIA Images and their Processing}

The major observational data we use came from SDO/AIA. The temperature response functions for the EUV channels of AIA were presented by Boerner et al. (2012), Lemen et al. (2012), and Downs et al. (2012). We use the well-known 171 and $193 \AA$ channels sensitive to normal coronal temperatures. The temperature response of the $211 \AA$ channel resembles the major peak of the $193 \AA$ channel shifted to 2 MK but lacks a minor high-temperature peak. The 94 and $131 \AA$ channels have two temperature sensitivity windows. The lower-temperature windows are sensitive to normal coronal temperatures, and the higher-temperature those have peaks at 6.3 and $10 \mathrm{MK}$, respectively. We also use the $304 \AA$ channel, which is sensitive to temperatures around $5 \times 10^{4} \mathrm{~K}$, with a lesser contribution from hotter plasmas around 1.8 MK.

Due to the location of the active region close to the limb, the erupting features were observed by AIA against the off-limb background. It has a considerable diffuse component, whose brightness is maximum at the limb and decreases with height. This diffuse background substantially reduces the contrast of the erupting features. In addition, their brightness, $I$, dramatically decreases in their expansion; with a conserved number of emitting particles, the brightness depends on a linear size, $r$, as $I \propto r^{-5}$ (Uralov et al., 2014, Grechnev et al., 2015). Such widely used ways as subtracting of an earlier image or dividing by it do not reveal static features and contain traces of the base image.

We have computed the radial brightness distributions from averages over ten pre-event images in each AIA channel using a ring scanning with an increasing radius (see Kochanov et al., 2013). This way is still not perfect because of strong differences between the low-latitude corona and regions above polar coronal holes. Nevertheless, the azimuthally averaged distributions allowed us to considerably enhance the appearance of off-limb features.

Subtraction of these background distributions enhances the contrast of offlimb features. Dividing by these distributions compensates for the upwards brightness decrease (probably, a similar way was used by Ma et al. (2011)). We use various combinations of both these ways.

\subsection{Kinematic Measurements}

Properties of eruptive structures and the causal relations between the underlying processes can be recognized from their kinematics, which is basically inferred 
from distance-time measurements. An obvious straightforward way to find the velocity and acceleration is the differentiation of the experimentally measured distance-time points. However, the measurements of an eruptive feature, which is usually faint relative to associated flare emission, are complicated by a rapid decrease of its brightness or opacity that leads to considerable uncertainties. The irregular appearance of the measured feature in the images causes a scatter of the inferred velocities and accelerations. Even the modern elaborations of the measurement techniques based on the direct differentiation of the experimental distance-time points (e.g., Vršnak et al., 2007, Temmer et al., 2010) do not overcome this difficulty completely, because the difference between the measured and actual position is always unknown.

An alternative approach is based on the fitting an analytic function to the measurements. Its major advantage is that the kinematical plots are calculated by means of the integration or differentiation of the analytic fit, thus providing a smooth outcome, rather than the differentiation of the measurements, that gives an intrinsically scattered result. If the kinematics of an analyzed feature is basically understood and described theoretically, then the problem is to compute the parameters of the corresponding analytic function.

Warmuth et al. (2001) proposed that fast Moreton waves observed in the $\mathrm{H} \alpha$ line and considerably slower "EIT waves" observed in EUV at larger distances were due to the same decelerating fast-mode disturbances. To fit their propagation, the authors attempted to use a 2nd-order polynomial and a power-law fit. Grechnev et al. (2008b) pointed out that a freely propagating blast-wave-like shock, which spent its energy to sweep up the plasma with a radial powerlaw density falloff, $n(x) \propto x^{-\delta}$ ( $x$ is the distance from the eruption center), and extrude it from the volume it occupied previously, indeed had a powerlaw kinematics, $x(t) \propto t^{2 /(5-\delta)} v s$. time $t$. Practically, we estimate the wave onset time, $t_{0}$ (e.g., from the analysis of a type II burst or from distance-time measurements), and, referring to the distance from the eruption center to one of the measured wave fronts, $x_{1}$, at time $t_{1}$, adjust $\delta$ to reach a best fit of the wave trajectory

$$
x(t)=x_{1}\left[\left(t-t_{0}\right) /\left(t-t_{1}\right)\right]^{2 /(5-\delta)} .
$$

This simple approximation satisfactorily fits various wave signatures such as "EUV waves", type II bursts, and leading edges of fast CMEs (see, e.g., Grechnev et al.] 2011b, 2011a, 2013a). The power-law density model, $n(h)=n_{0}\left(h / h_{0}\right)^{-\delta}$, with $h$ being the height from the photosphere, $n_{0}=4.1 \times 10^{8} \mathrm{~cm}^{-3}, h_{0}=100 \mathrm{Mm}$, and $\delta=2.6$ is close to the equatorial Saito model (Saito, 1970) at $h \geq 260 \mathrm{Mm}$ within $\pm 30 \%$, providing a steeper density falloff at lesser heights. For the usage of the power-law fit in the analyses of imaging data and dynamic radio spectra see Grechnev et al. (2014b).

For the kinematics of CME structures, which have been completely formed and acquired maximum accelerations, we use analytic equations obtained in a self-similar approach (Uralov, Grechnev, and Hudson, 2005. Grechnev et al., 2008b) This approximation is based on the fact that the relation between the propelling and retarding forces (magnetic pressure and tension, plasma pressure, 
and gravity) applied to any element of the expanding CME, established after the impulsive acceleration stage, decreases by the same factor with an increase of the distance from the expansion center (e.g., Low, 1982). This approximation applies, as long as the aerodynamic drag of the solar wind has a minor importance, i.e., until the regime of the plasma extrusion by the CME bubble changes to the regime of the plasma flow around its outer surface. The self-similar equations are complex and resemble hyperbolic functions in behavior (Grechnev et al., 2014b).

The kinematics of eruptive features during the impulsive acceleration stage has not yet been well understood. In this case, a more or less suitable analytic function can be chosen from considerations based on the properties, which have already been established. One knows a priori that the initial velocity is typically small or zero, the final velocity is nearly constant, and the acceleration occurs impulsively within a certain time. Considerable short-time variations of the acceleration and velocity are not expected well after the impulsive acceleration stage. The particular shape of the acceleration pulse does not substantially affect the distance-time plot because of the double integration. A bell-shaped acceleration pulse meets these speculations. If a real distance-time plot considerably deviates from the kinematics described with a single acceleration pulse, then a combination of two (or more) pulses can be used.

This approach has been successfully used in several studies by different authors (e.g., Gallagher, Lawrence, and Dennis, 2003; Sheeley, Warren, and Wang, 2007. Wang, Zhang, and Shen, 2009) as well as in our studies (Grechnev et al. 2011b, 2013a, 2014a; Alissandrakis et al., 2013). In our technique, the results of the fit are used as a starting estimate of the parameters of the acceleration, and then they are optimized to outline the eruption in a best way. Our ultimate criterion is to follow the analyzed feature as closely as possible in all of the images. The major source of the errors is the uncertainty in following the same moving feature, whose visibility progressively decreases.

The most important issue in our present study is the kinematics of the flux rope, which was even difficult to detect. We fit the measured projected heights of the flux rope, $h(t)$, with a smooth function that accounts for its acceleration and deceleration phases. We modified equation (1) from Sheeley, Warren, and Wang (2007) to the following two-pulse form:

$$
\begin{array}{r}
h(t)=h\left(t_{1}\right)+\frac{1}{2}\left(v_{\mathrm{f}}^{+}+v_{0}^{+}\right)\left(t-t_{1}\right)+\frac{1}{2}\left(v_{\mathrm{f}}^{+}-v_{0}^{+}\right) \tau_{1} \ln \left[\cosh \left(\frac{t-t_{1}}{\tau_{1}}\right)\right]+ \\
h\left(t_{2}\right)+\frac{1}{2}\left(v_{\mathrm{f}}^{-}+v_{0}^{-}\right)\left(t-t_{2}\right)+\frac{1}{2}\left(v_{\mathrm{f}}^{-}-v_{0}^{-}\right) \tau_{2} \ln \left[\cosh \left(\frac{t-t_{2}}{\tau_{2}}\right)\right] .
\end{array}
$$

Here $v_{0}$ and $v_{\mathrm{f}}$ are the initial and final asymptotic values of velocity for the acceleration $(+)$ and deceleration (-) pulses, $t_{1}$ and $t_{2}$ are the acceleration and deceleration center times, $h\left(t_{1}\right)$ and $h\left(t_{2}\right)$ are the corresponding heights, and $\tau_{1}$ and $\tau_{2}$ are the timescales of the acceleration and deceleration. The corresponding velocity, $v(t)$, is

$$
v(t)=\frac{1}{2}\left(v_{\mathrm{f}}^{+}+v_{0}^{+}\right)+\frac{1}{2}\left(v_{\mathrm{f}}^{+}-v_{0}^{+}\right) \tanh \left(\frac{t-t_{1}}{\tau_{1}}\right)+
$$




$$
\frac{1}{2}\left(v_{\mathrm{f}}^{-}+v_{0}^{-}\right)+\frac{1}{2}\left(v_{\mathrm{f}}^{-}-v_{0}^{-}\right) \tanh \left(\frac{t-t_{2}}{\tau_{2}}\right) .
$$

The acceleration profile, $a(t)$, with the contributions from the two pulses is

$$
a(t)=\frac{v_{\mathrm{f}}^{+}-v_{0}^{+}}{2 \tau_{1}}\left[1-\tanh ^{2}\left(\frac{t-t_{1}}{\tau_{1}}\right)\right]+\frac{v_{\mathrm{f}}^{-}-v_{0}^{-}}{2 \tau_{2}}\left[1-\tanh ^{2}\left(\frac{t-t_{2}}{\tau_{2}}\right)\right] .
$$

We fit the analytic function $h(t)$ to the measured data using the LevenbergMarquardt least-squares minimization (Levenberg, 1944: Marquardt, 1963) implemented by C.B. Markwardt in the SolarSoft MPFIT package. To evaluate the confidence intervals of the resulting fit, i.e., to estimate the influence of the measurement errors on the inferred quantities (the velocity and acceleration profiles), we use a technique similar to the parametric bootstrap method. Numerous simulation runs are carried out to produce a large number of data sets, in which the measured data points are displaced by normally distributed pseudo-random numbers with $\sigma$ corresponding to the measurement errors. Then we calculate the variance for the parameters of the fit. In this way, we also monitor the stability of the fit against noisy data.

The whole set of the parameters used in our fit is redundant, while $v_{\mathrm{f}}^{+}=v_{0}^{-}$ appears to be sufficient. We have to keep all of them to ensure a stable behavior of our fitting software in its present implementation.

To study the evolution of an expanding feature, we adjust the field of view of the images to keep its visible size fixed according to the measured kinematics (Grechnev et al., 2014b, 2015). Residual trends can be detected in looking at a movie composed from the resized images to improve the parameters of the fit at the next iteration.

\subsection{Estimations of Plasma Parameters}

Important information about eruptive structures and their properties can be obtained from their temperature and emission measure (EM). Qualitative judgments about the temperatures of coronal structures observed by SDO/AIA can be inferred from their appearance in different channels based on their temperature response functions (Boerner et al., 2012 Lemen et al., 2012, Downs et al., 2012) If EM of an emitting structure is known, then, with a size found from the images, its density and mass can be estimated.

The most general way is the inversion of the differential EM (DEM) of the structures observed nearly simultaneously in different AIA channels. Actually, the image in each next AIA channel is produced $12 \mathrm{~s}$ after the preceding one. Eruptive features of our interest can acquire high speeds that causes their appreciable displacements in the AIA images even during the relatively short time intervals between them. To reduce the errors due to this effect, we resize the images produced in all of the AIA channels according to the measured kinematics of a feature in question, as described in the preceding section. Practically, we take in this case two sets of the AIA images, one observed one time step before the time of interest, and the second - the next set, resize each image according to its observation time, and then interpolate each pair of the images in each 


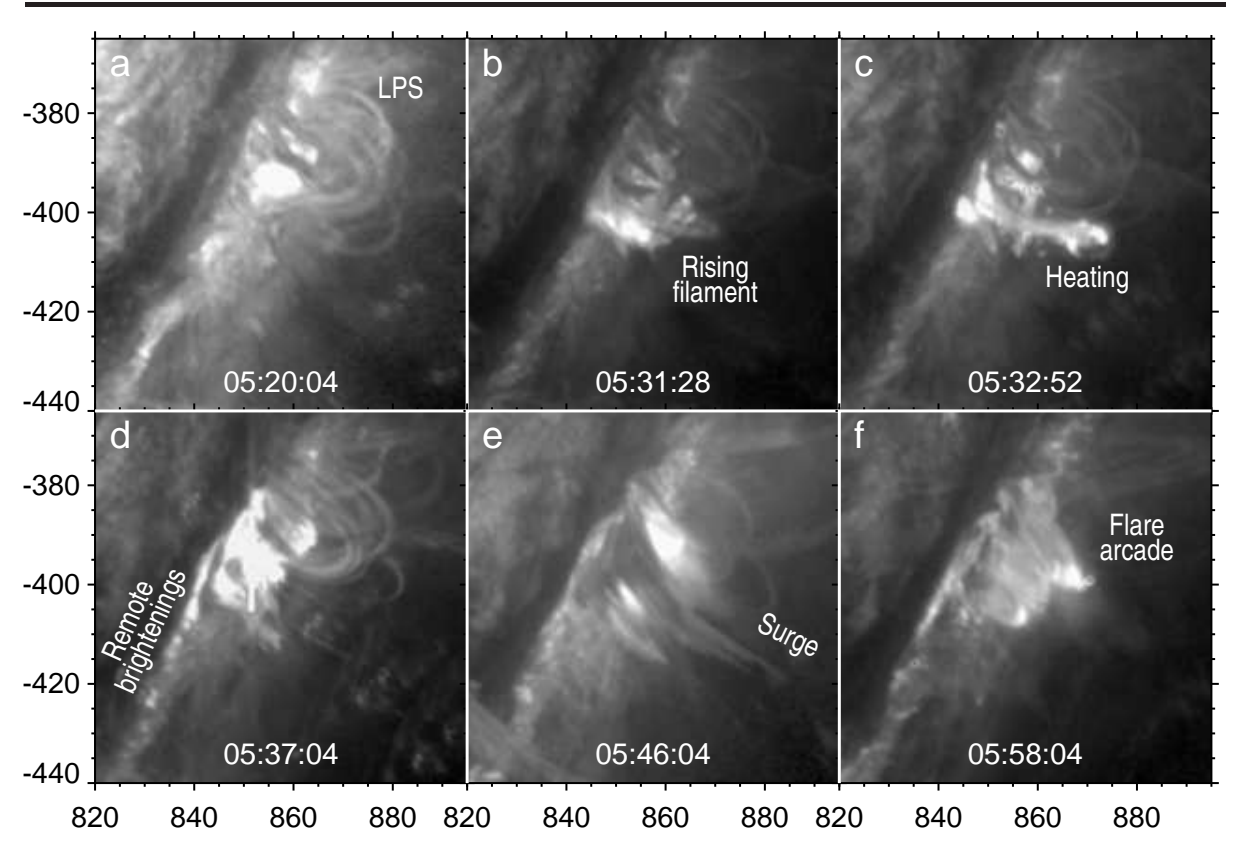

Figure 1. Activity in the low corona throughout the event observed in SDO/AIA $304 \AA$ images. Label 'LPS' in panel (a) denotes a loop prominence system. The axes show hereafter the coordinates in arcsec from the solar disk center.

channel to the required time. This procedure improves the co-registration of the images.

In our analysis we use for reliability two different ways. These are the regularized inversion technique and software developed by Hannah and Kontar (2012. hereafter HK for brevity) and a faster algorithm developed by Plowman, Kankelborg, and Martens| (2013 hereafter PKM). Then we compare the results produced with the HK and PKM algorithms.

\section{Geometry and Orientation}

Figure 1 presents some episodes of the event observed in the SDO/AIA $304 \AA$ channel. In a pre-event configuration in Figure 1 $1 \mathrm{~h}$, a loop prominence system (LPS) is considerably inclined to the line of sight. LPSs are known to be located above the main magnetic polarity inversion (neutral) lines.

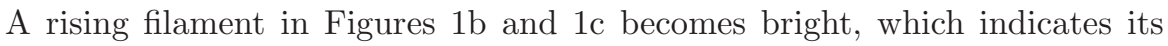
heating. Then the hot top of the filament becomes transparent and disappears later on. In Figure 1, remote compact bright kernels intermittently appear and fade, being arranged in a direction nearly parallel to the axis of the LPS. The plane of a dark surge in Figure 1 and the orientation of the flare arcade in Figure 1f also correspond to the inclined direction of the LPS. 


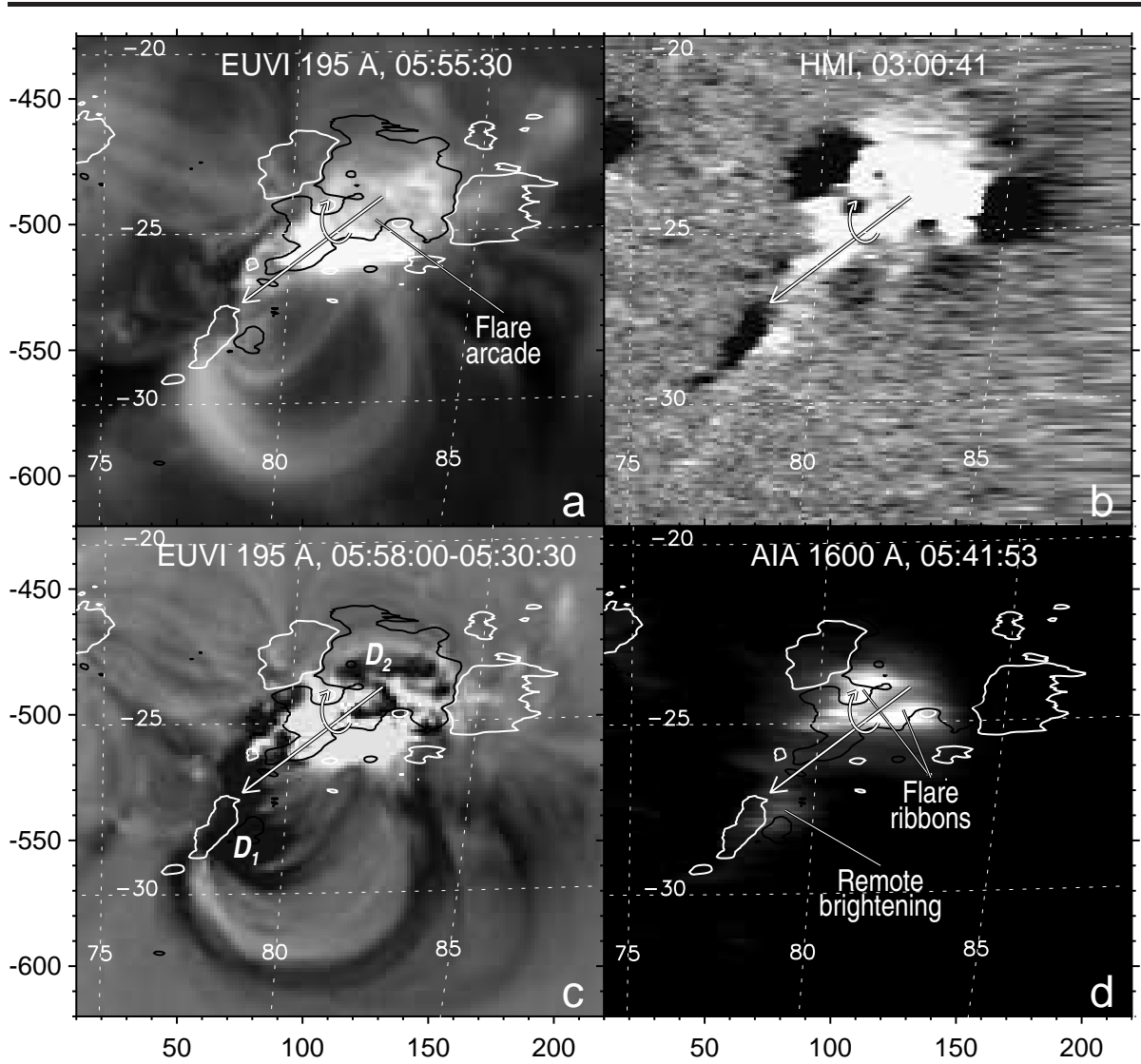

Figure 2. Flare configuration observed from STEREO-A along with contours of an SDO/HMI magnetogram transformed to this viewing direction. The contour levels correspond to $-35 \mathrm{G}$ (white) and $+35 \mathrm{G}$ (black) in the magnetogram smoothed with a 5-pixel boxcar. (a) Flare arcade in an EUVI $195 \AA$ image. (b) HMI magnetogram, rotated to match the view from STEREO-A, within a range of \pm 50 G. (c) Dimmed regions in an EUVI $195 \AA$ Aifference image. The dimming regions presumably associated with the footprints of the flux rope are denoted $D_{1}$ and $D_{2}$. (d) Flare ribbons and remote brightening in an SDO/AIA $1600 \AA$ image transformed to the viewing direction from STEREO-A. The straight arrow indicates the orientation of the axial magnetic field in the flux rope $\left(-37^{\circ}\right)$. The round arrow shows the direction of the magnetic field in the arcade. The heliographic grid corresponds to viewing from Earth.

\subsection{Overall Configuration}

Complementary observations from the STEREO-A vantage point make the overall configuration clearer. Figure2 compares the STEREO-A/EUVI $195 \AA \AA$ images with an SDO/HMI line-of-sight magnetogram (Figure 2b) and the flare ribbons in the AIA $1600 \AA$ image (Figure $2 \mathrm{~d}$ ). The SDO data were transformed to the viewing direction from STEREO-A. A strong projection shrinkage of the near-the-limb flare site reduces the quality of the transformed SDO images. The westernmost part of the magnetogram appears with an inverted polarity, which is most likely an artifact due to projection effects on magnetic field inclined to the line of sight, which is a common feature in magnetograms close to the limb. 
The orientation of the flare arcade (partly saturated) in a late EUVI image in Figure 2a corresponds to the flare ribbons in a transformed $1600 \AA$ AIA image in Figure 2 $\mathrm{d}$. The ribbons must be separated by the magnetic neutral line, whose direction is shown by the straight tilted arrow, which corresponds to the orientation of the LPS and arcade in Figure 1

A difference image in Figure 2 2 reveals the regions of dimming, some of which are probably due to displacements of the loops visible in Figure 2 a or eruption of their neighbors. The core dimmings $\mathrm{D}_{1}$ and $\mathrm{D}_{2}$ might be associated with the footprints of an erupted flux rope (Hudson and Webb, 1997; Sterling and Hudson, 1997; Webb et al., 2000; Mandrini et al., 2005). This assumption is confirmed by a remote brightening in $1600 \AA$ within $\mathrm{D}_{1}$ in Figure $2 \mathrm{~d}$ (cf. Figure 11). A conjugate footpoint of the flux rope must be within an opposite polarity; region $\mathrm{D}_{2}$ meets this requirement. The direction of the flux rope's azimuthal magnetic field (the round arrow) should correspond to the flare arcade, being prompted by the magnetogram in Figure $2 \mathrm{~b}$, although considerably distorted.

The observations indicate that the flux rope was compact, with a length comparable to its width. Its axis was initially tilted by $\approx-37^{\circ}$ to the East.

\subsection{CME Lift-off}

The eruption produced a CME, whose lift-off was observed by SDO/AIA in different-temperature channels. They reveal various coronal structures (see also the AIA_131_171_loops.mpg movie in the electronic supplementary material).

The $171 \AA$ channel is sensitive to coronal features of relatively low temperatures. Figure 3 shows some episodes of the CME development in the AIA $171 \AA$ images starting from the pre-event configuration in Figure 3 a. The orientation of the pre-eruptive arcade here corresponds to the inferred tilt of the flux rope's axis of $-37^{\circ}$. The black arc outlines the top of the visible set of the loops.

As the developing CME lifts off, the arcade loops get involved in the expansion from below upwards (Patsourakos, Vourlidas, and Stenborg, 2010; Eselevich and Eselevich, 2013). The rising lower loops press the overlying ones, and finally all of them apparently merge into a very thin, nearly circular rim. This process is addressed in Section 5] Most authors, who studied this event previously, invoked a traditional assumption of the identity of the flux rope with the CME cavity, and related its outer boundary to the visible rim. The rim was considered as a cross section of the flux rope viewed nearly along its axis.

However, the rim in Figure 3 resembles a balloon with a thin skin or a soap bubble. With the estimated orientation of the flux rope, its cylindrical shape would contradict the appearance of the rim. Even though a possible rotation of the flux rope around the continuation of the solar radius is not excluded, the appearance of the rim corresponds to a roughly spheroidal or pyriform CME bubble rather than a long cylinder viewed nearly along its axis.

The curvature of the black arc outlining the top of the arcade, which transformed into the rim, decreased in Figures $3 \mathrm{a}-3 \mathrm{~d}$. This effect was revealed by Patsourakos, Vourlidas, and Stenborg (2010). Then the curvature gradually increased again, as the movie demonstrates. A bright kernel inside the bubble (visible also in 193 and $211 \AA$ ) might be a largest-opacity flux-rope center. 


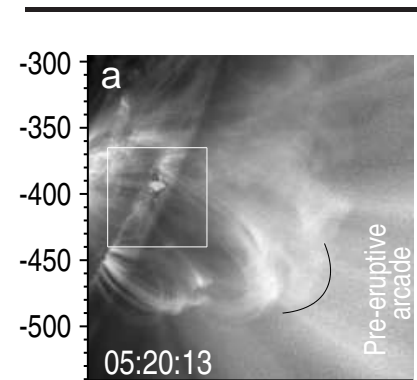

85090095010001050

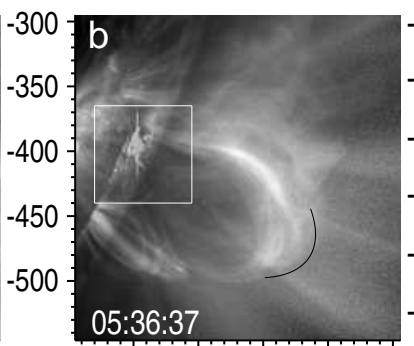

85090095010001050

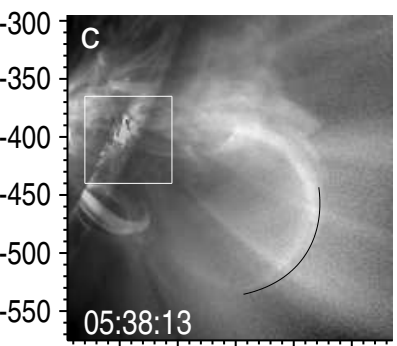

85090095010001050
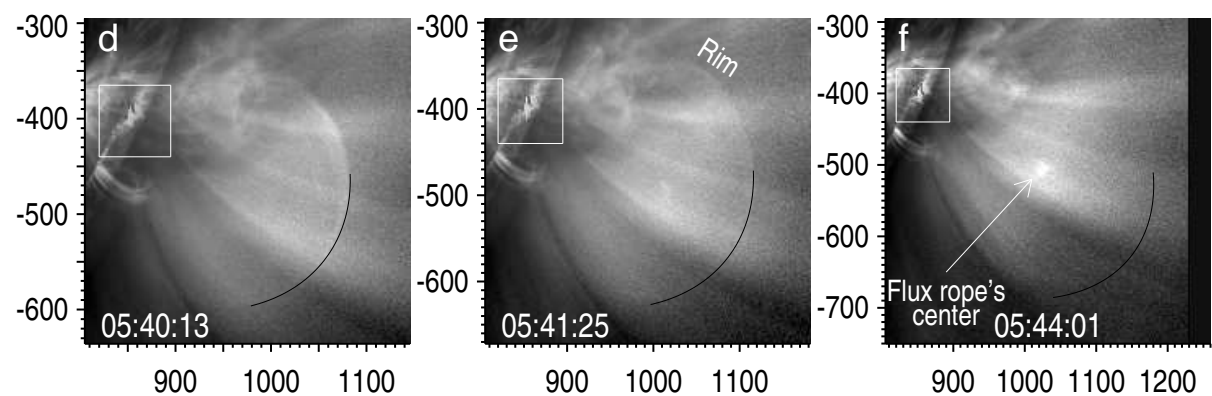

Figure 3. Development of the CME bubble in SDO/AIA $171 \AA$ images. The images are progressively resized to keep the size of the outermost loop outlined by the black arc unchanged. The white frame corresponds to the field of view in Figure 1

The $211 \AA$ AIA images with a reduced background in Figure 4 reveal a low-brightness higher-temperature environment of the AR consisting of closed structures. They were higher and had different orientations from the loops visible in $171 \AA$. The loops in $211 \AA$ are faint in the pre-event image in Figure $4 \mathrm{a}$ and become discernible later (e.g., in Figure 4e). The different orientations of the loops below the rim and above it suggest its association with a separatrix surface.

A separatrix surface prevents mixing magnetic structures, which belong to different magnetic domains isolated by this surface. The expanding rim associated with a separatrix surface limits the expansion of the loops below and sweeps up the structures above, leaving a rarefied volume behind. No dimming is pronounced in Figure 4, because we only reduced the structureless coronal background in front of the bubble and behind it, without subtracting any preceding image.

The black arc outlines the top of the rim, and the white arc, whose radius is $90^{\prime \prime}$ larger, acceptably matches the outer edge of the pileup, which probably becomes the leading edge of the future CME later on. The expansion velocities of the rim and pileup from 05:40 to 05:44 were not much different.

North of the AR, a ray-like feature resembling a small streamer is denoted in Figure 4a. Presumably at its base, a quadrupole configuration is present in STEREO-A/EUVI $195 \AA$ images about 100" west of the AR. This site is a candidate for a source region of a type II radio burst discussed in Section 6 . 


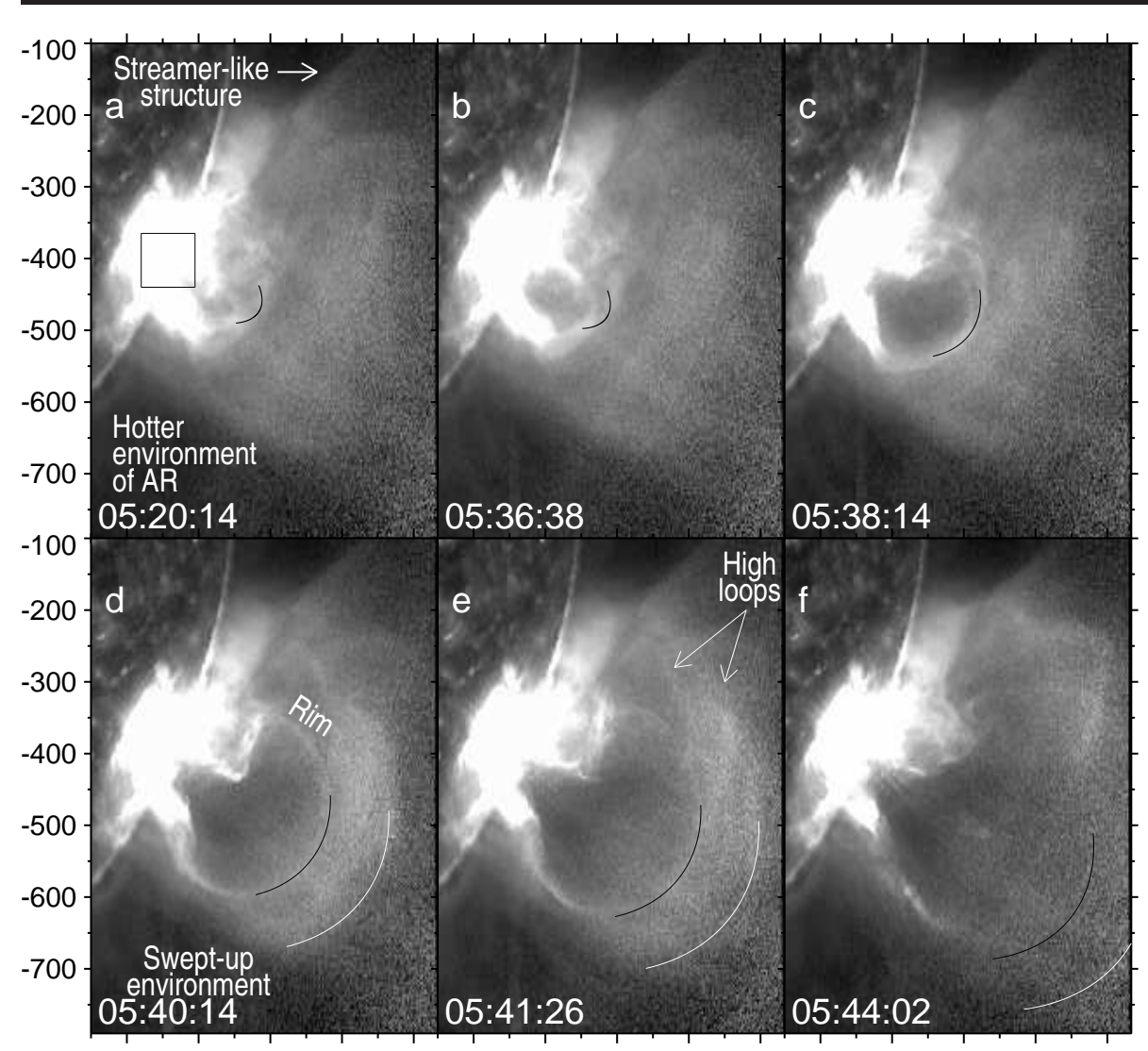

800900100011001200800900100011001200800900100011001200

Figure 4. Development of the CME bubble in SDO/AIA $211 \AA$ images. The black arc corresponds to the arc in Figure 3 The white arc in panels (d) - (f) is $90^{\prime \prime}$ farther. The expanding rim sweeps up the bright structures enclosed between the arcs. The black frame in panel (a) corresponds to the field of view in Figure 1

\section{Flux Rope}

\subsection{Genesis and Expansion}

According to Patsourakos, Vourlidas, and Stenborg (2010), the CME lift-off was possibly triggered by the eruption of a tiny filament $(\lesssim 20 \mathrm{Mm})$. It is shown in Figures 10 and 1,. We start to search for the elusive flux rope from the activation of the filament observed in $131 \AA$.

The initial dark filament (Figure $5 \mathrm{a}$ ) activated in Figures $5 \mathrm{~b}-5 \mathrm{~d}$. The history of the event can be followed in Figure 5rom the two soft X-ray (SXR) GOES channels and a light curve computed from the $131 \AA$ images over a $153^{\prime \prime} \times 153^{\prime \prime}$ region centered at $\left(855^{\prime \prime},-396^{\prime \prime}\right)$ to encompass the flare site. The gray vertical bars in Figure 5 , represent the intervals, in which the images were averaged. The $131 \AA$ light curve reveals a long gradual rise of the emission during 05:00-05:30 


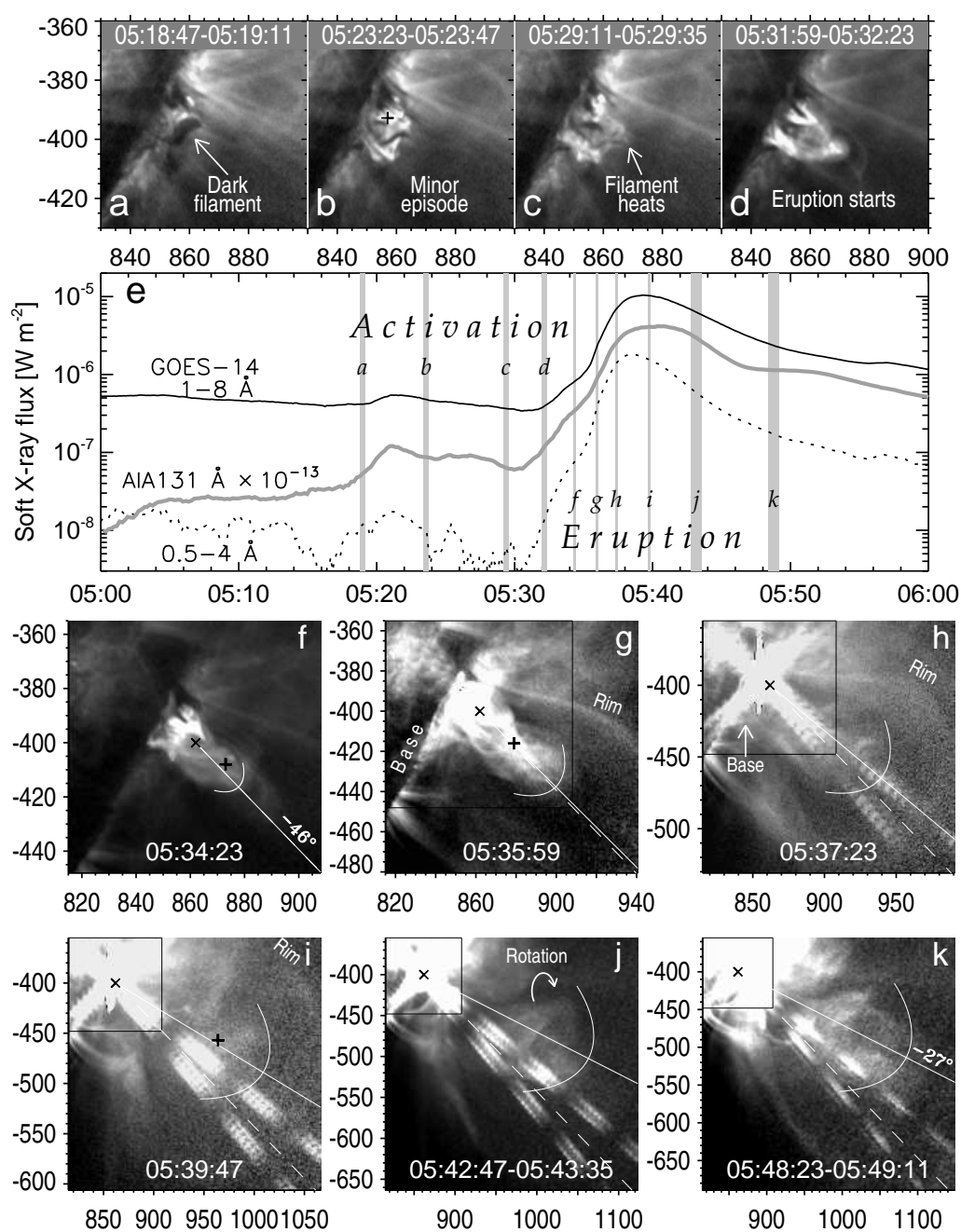

Figure 5. (a-d) Filament activation in averaged AIA $131 \AA$ images. (e) GOES flux in $1-8 \AA$ (black solid) and 0.5-4 $\AA$ (dotted) and a light curve computed from the $131 \AA$ images over the flare region (gray). (f-k) Erupting flux rope in $131 \AA$ images resized to keep its extent. The arcs outline its top. The solid line goes from the origin of the measurements (slanted cross) and the flux rope center. The dashed line shows its initial orientation $-46^{\circ}$ from the west, the straight crosses in panels (b), (f), (g), and (i) denote the positions, for which DEM in Figure 7 was computed. The black frame denotes the field of view in panel (f). The images in panels (j) and (k) were averaged in the specified intervals after resizing. The coordinates indicate position in arcsec from disk center at the middle of the averaging intervals.

from the filament or its environment, indicating heating processes. This rise is not present in the GOES data, which respond to the whole Sun's emission.

The activated part of the filament in Figure 5 b brightens up during a minor episode of 05:20-05:24 before the major eruption, supporting its heating. A similar appearance of the brightened filament in different AIA channels suggests a wide range of plasma temperatures in its body. A response to this episode 
in 1-8 $\AA$ and even in 0.8-4 $\AA$ (although marginal) indicates that the filament brightening could be caught in the high-temperature window of the $131 \AA$ channel. The top of the filament in Figure 55 becomes bright and transparent. The eruption starts in Figure $5 \mathrm{~d}$.

The average temperature of the brightened filament estimated from the two GOES channels is $\approx 6.6 \mathrm{MK}$. All of the estimates indicate that the $131 \AA$ images are most promising to reveal the disappearing top part of the erupting filament, its possible relation to the flux rope, and the flux rope itself. The high-temperature window of the $94 \AA$ channel could also be appropriate, but its sensitivity is considerably lower. We therefore focus on the $131 \AA$ images.

A sharp increase of the emission after 05:30 (Figure 5 e) caused strong overexposure effects such as saturation, blooming, and oblique diffraction patterns. Nevertheless, the image processing described in Section 2.1 allowed us to detect an erupting flux rope in $131 \AA$ shown in Figures 5 5 - 5 k and the flux_rope_131.mpg movie. The images are resized to fix the visible extent of the flux rope using the kinematical measurements described in the next section.

The heated filament body in Figure $5 \mathrm{H}$ transforms into an erupting bundle of twisted loops in Figure 5. Faint outermost loops disappear soon. The bundle rapidly expands along the dashed line inside the rim, as a decreasing black frame (field of view in Figure 5) indicates. Several threadlike loops are rooted at the base denoted in Figures 55 and $5 \mathrm{~h}$. As the rope rises, its Earth-facing base expands southeast, producing the remote birghtenings in Figures $1 \mathrm{~d}$ and $2 \mathrm{~d}$.

The direction of the lift-off (solid line) gradually turns aside by $\approx 20^{\circ}$. The flux rope rotates (see the circular arrow in Figure 5 and the movie). More loops still erupt and join the flux rope in latest images. The latest visible loops are apparently injected into the northern part of the flux rope's bottom.

This sequence of events is faintly visible in $131 \AA$ (characteristic temperature $10 \mathrm{MK}$ ). To reveal the flux rope in Figure 5 we had to average a few images within the specified intervals. The flux rope can also be detected in $94 \AA$ (6.3 MK), but still poorer. These circumstances indicate that its temperature was around $10 \mathrm{MK}$.

\subsection{Kinematics}

We used a few different ways to measure the kinematics of the expanding flux rope. The major difficulty was its decreasing brightness, which became comparable with noise in the images, so that the flux rope eventually disappeared. To get a hint at its final speed, we analyzed the images of the SOHO's Large Angle and Spectroscopic Coronagraph (LASCO; Brueckner et al., 1995). The flux-rope's center expanded in LASCO/C2 images after 09:30 with a speed of $\approx 52 \mathrm{~km} \mathrm{~s}^{-1}$, while the speed of its top was considerably less than the asymptotic speed of the frontal structure, $190 \mathrm{~km} \mathrm{~s}^{-1}$ (Figure 13 in Section 5.2). The top of the flux rope was $30-40 \%$ ahead of its center visible in late AIA $193 \AA$ images. These speculations indicate a final speed of $60-80 \mathrm{~km} \mathrm{~s}^{-1}$ for the flux rope's top.

Using the results of preliminary measurements, we produced a movie, in which we scaled the field of view to compensate for the expansion of the flux rope. The movie shows it clearer. If the visible size of the flux rope in the movie still varied, 


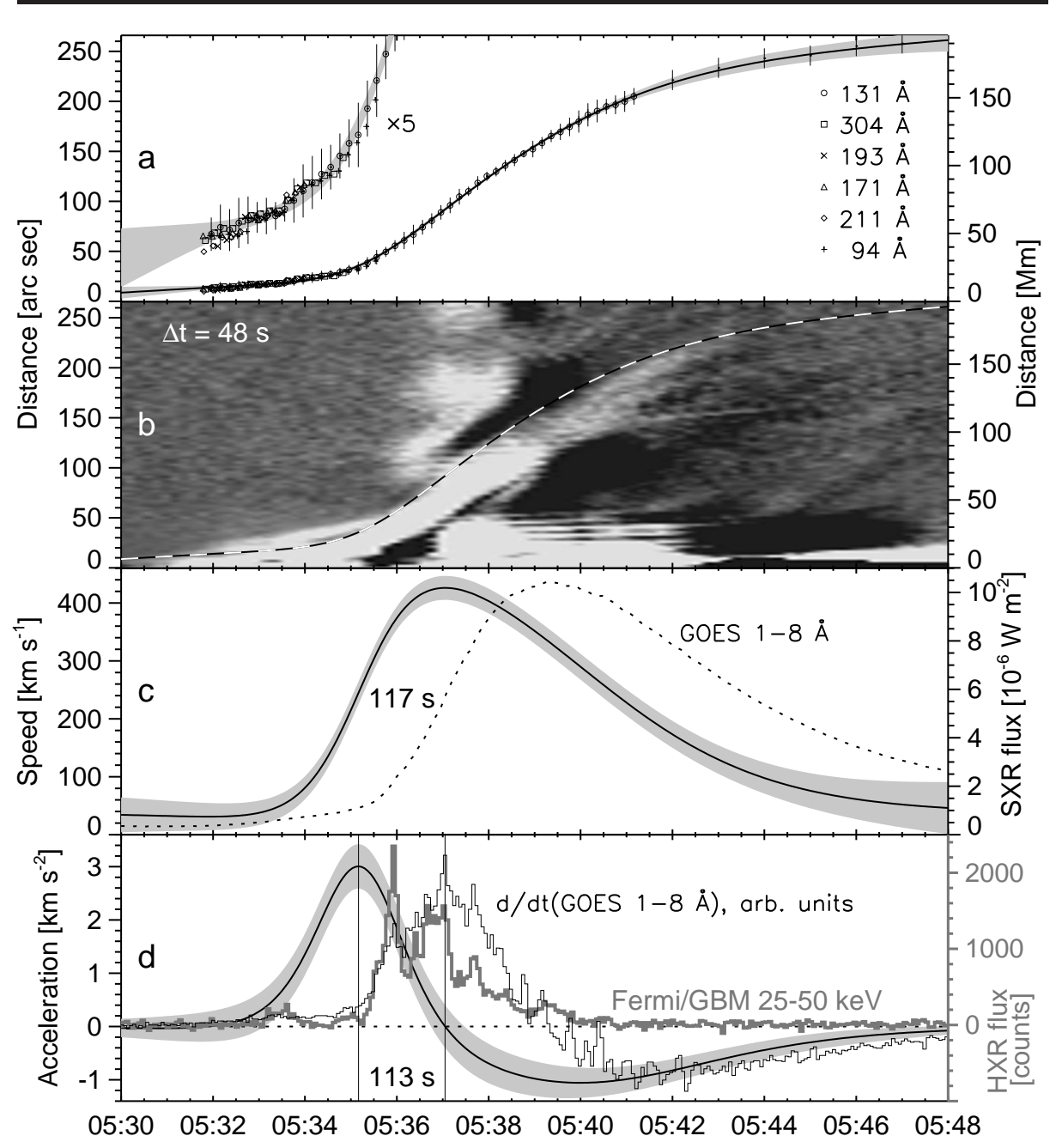

Figure 6. Kinematics of the flux rope. (a) Direct manual distance-time measurements (symbols) and the analytic fit (solid line). The initial part enlarged by a factor of five is also shown. The bars represent the errors of measurements from the $131 \AA$ images estimated subjectively. The shadings in panels (a), (c), and (d) represent the uncertainties evaluated by the parametric fit. (b) One-dimensional time history of the flux rope's lift-off in $131 \AA$ running-difference images separated by $48 \mathrm{~s}$. The dashed line represents the analytic fit from panel (a). (c) Velocity-time plot computed from the analytic fit (solid) along with a GOES flux (dotted). (d) Acceleration of the flux rope (black), hard X-ray burst (gray), and the derivative of the GOES flux (thin).

then we refined our measurements and repeated the attempt. These various ways converged into the results presented in Figure 6

The starting manual distance-time measurements are presented in Figure 6 a. The heights are related to the varying direction mentioned in the preceding section. The correspondence between the tops of the filament measured from six 
AIA channels while it was detectable (the magnified initial part) and the flux rope in the $131 \AA$ images confirms their genetic relation.

We also manually adjusted an acceleration profile consisting of a positive pulse followed by a negative one (Grechnev et al., 2015). Finally, elaborating this approach, we developed the automatic fit described in Section 2.2. The black curve in Figure 6 a is its result. The shading represents the calculated uncertainties.

The fit is superimposed on a time-history image in Figure [6 (similar to the slit images used by Ma et al., 2011, see also Alissandrakis et al., 2013: Grechnev et al., 2014a). Each column of this image is a spatial profile computed as averages over a 12-pixel $\left(7.2^{\prime \prime}\right)$ wide slice extracted from a running-difference $131 \AA$ image with a cadence of $48 \mathrm{~s}$. The instant orientations of the slices follow the turning flux rope. The expanding flux rope appears in this image as a bright strip. The fit should be its upper envelope. A bright feature visible above the fit from 05:31:30

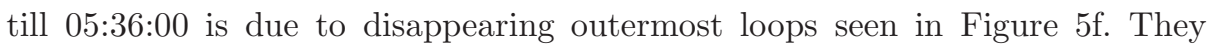
started expanding earlier and had a nearly constant speed.

Figure 6. shows the velocity-time plot computed from the analytic fit, the uncertainties (shaded), and the GOES SXR flux. The flux-rope velocity started to sharply rise at 05:33, exceeded $400 \mathrm{~km} \mathrm{~s}^{-1}$ at $05: 37$, and then decreased to $\approx 50 \mathrm{~km} \mathrm{~s}^{-1}$. The SXR flux is similar to the velocity plot, but lags behind it by $117 \mathrm{~s}$. The similarity of the rise phases is expected in the scenario of Hirayama (1974), where flare processes are driven by the erupting filament. The similarity of the declining-phase parts might be due to expansion of the flare arcade in the wake of the CME (Livshits and Badalyan, 2004).

Figure 6 $\mathrm{d}$ shows the computed acceleration plot (thick black) with uncertainties, hard X-ray (HXR) flux from the Gamma-Ray Burst Monitor of the Fermi Gamma-ray Space Telescope (Fermi/GBM; Meegan et al., 2009) and reconstructed within the 25-50 keV energy band for this burst (thick gray); and the derivative of the GOES flux (thin). The flux rope underwent a strong acceleration up to $3 \mathrm{~km} \mathrm{~s}^{-2} \approx 11 g_{\odot}$ at $05: 35: 10 ; g_{\odot}=274 \mathrm{~m} \mathrm{~s}^{-2}$ is the solar gravity acceleration at the photospheric level. The acceleration changed to a longer deceleration, which reached $-1 \mathrm{~km} \mathrm{~s}^{-2}$ at about 05:40:00.

The sharply accelerating flux rope must have produced a strong wavelike disturbance. It must propagate omnidirectionally, initially with a fast-mode speed, $V_{\text {fast }}$. Typically, $V_{\text {fast }} \gtrsim 10^{3} \mathrm{~km} \mathrm{~s}^{-1}$ in the low corona above active regions.

The time resolution of the Fermi/GBM data of an enhanced spectral resolution we use is $4 \mathrm{~s}$. The time bins of the GOES-14 SXR data are $2 \mathrm{~s}$. The positive portion of the derivative of the SXR flux roughly resembles the HXR burst (the Neupert effect; (Neupert, 1968) and contains counterparts of most HXR features without a detailed correspondence. The derivative of the SXR flux is similar to the acceleration pulse, lagging behind by $113 \mathrm{~s}$. The lag of the HXR and microwave emissions by 1-2 minutes behind the acceleration of an eruptive structure seems to be a systematic phenomenon. We observed it previously in a few other events (Grechnev et al. 2011b, 2013a, 2015). 


\subsection{Differential Emission Measure}

We computed DEM from sets of AIA images produced nearly simultaneously in different channels (see Section 2.3). Figure 7 presents the results for four episodes of the flux-rope development: the minor episode of the filament heating in Figure 5 (05:21:13, Figure 7 $\mathrm{h}$ ), the early flux-rope appearance in Figure 5 (05:33:30, Figure 7b), is half-height acceleration in Figure 5b (05:35:30, Figure $7 \mathrm{f}$ ), and during the deceleration stage in Figure 5 (05:39:02, Figure 7d). The times and centers of the boxes, in which DEM was calculated, are listed in the upper-left corner of each panel. It was not possible to relate the calculations to the same part of the flux rope because of sharp changes in its shape and strong overexposure distortions of the AIA images. The observation times, for which DEM was computed, are not identical to the most representative images of the flux rope in Figure 5

The PKM profiles in the lower-temperature range in Figures $7 \mathrm{~b}$ and 7 , do not look perfect, possibly due to the strong instrumental distortions, while the HK profiles seem to be more plausible. Our major interest is related to the shaded high-temperature domain. Here both methods supplied similar results, differing quantitatively in the maximum DEM by factors of $3.4,2.9$, and 0.58 for the three times, respectively. The temperature of the flux rope progressively increased from $\approx 10 \mathrm{MK}$ at 05:33:30 to $12 \mathrm{MK}$ at 05:35:00 and then to $17 \mathrm{MK}$ at 05:39:02.

The visible widths of the flux rope at 05:35:00 and 05:39:02 were $d_{2} \approx 21^{\prime \prime}$ and $d_{3} \approx 120^{\prime \prime}$. If the total number of emitting particles inside the expanding volume was conserved, then the expected decrease of the brightness (i.e., DEM; Grechnev et al., 2015) should be $\left(d_{3} / d_{2}\right)^{5} \approx 6100$. Actually, the DEM decrease from 05:35:00 to 05:39:02 was much less, 1200 (HK method) and 230 (PKM method). A similar situation occurred also between 05:33:30 and 05:35:00.

The density and mass of the hot flux rope listed in Figures $7 \mathrm{~b}-7 \mathrm{~d}$ were estimated from its plane-of-the-sky width assuming the spherical shape of its upper part (similar in Figure 7 $7 \mathrm{~h}$ ). The ratios of their estimates with the HK and PKM methods are 2.1, 1.1, and 0.89 at the three times that seems to be reasonable, considering the faintness of the flux rope.

All of the estimates indicate that the mass of the flux rope considerable increased. Along with the increase of its temperature, this fact suggests an ongoing injection of hot plasma from the flaring region; otherwise, the temperature increase were challenging. As mentioned, the injection of high-temperature loops is indeed faintly visible at late stages of the eruption. The rotational momentum supplied by these non-centrally injected loops possibly caused the rotation of the flux rope indicated in Figure 5 and visible in the movie.

\section{Development of CME Structural Components and Their Later Expansion}

\subsection{CME Formation in EUV Images}

The phenomena observed during the CME lift-off in this event were analyzed by Patsourakos, Vourlidas, and Stenborg (2010); Eselevich and Eselevich (2013); and 


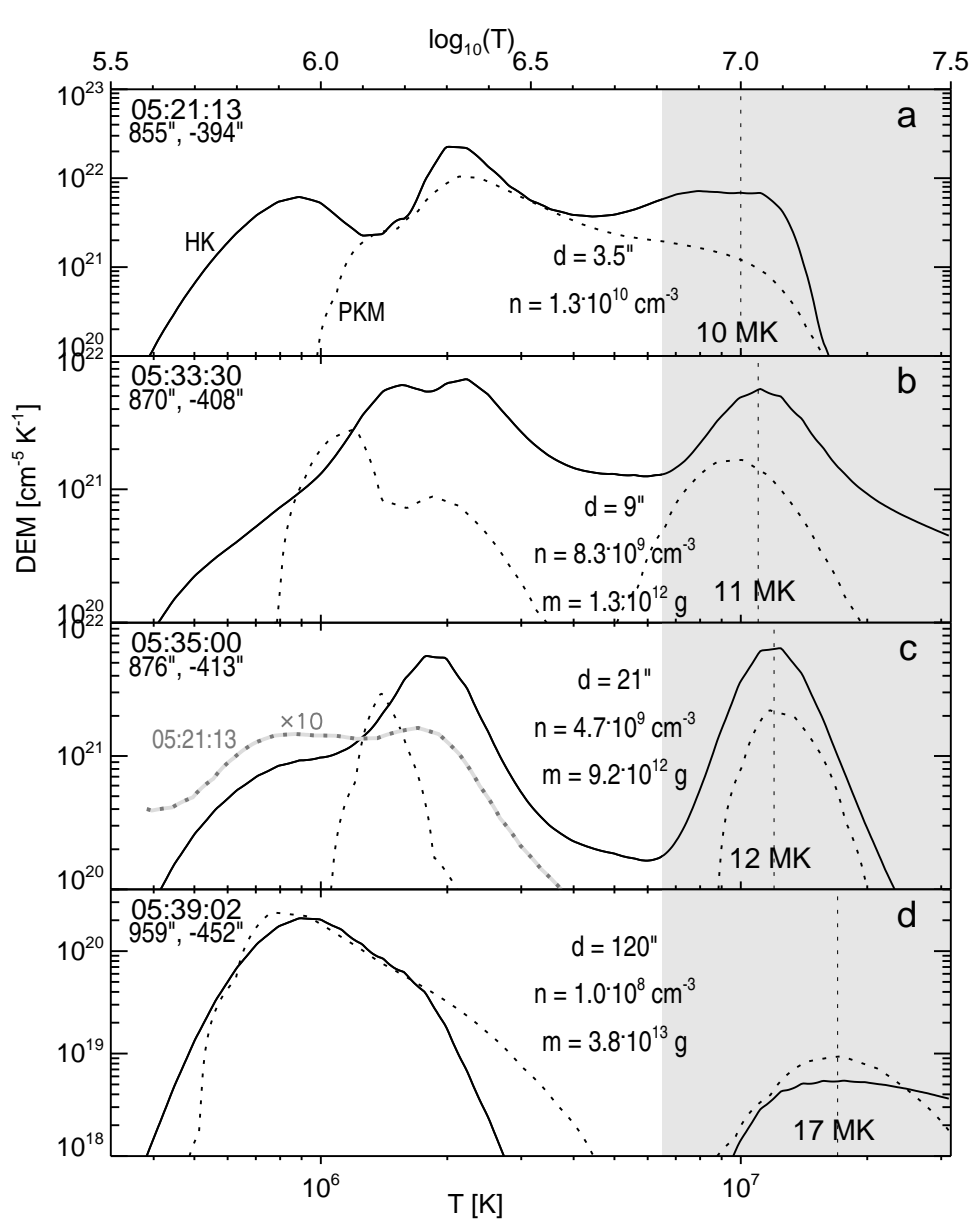

Figure 7. DEM temperature profiles in the heated filament during the minor filament heating episode at 05:21:13 (a) and the flux rope at 05:33:30 (b), 05:35:30 (c), and 05:39:02 (d). DEM was computed using the HK (solid) and PKM (dotted) methods. The plots are related to the regions, whose centers are denoted by the straight crosses in Figures $5 \mathrm{5}, 5 \mathbf{5}, \mathbf{5} \mathrm{g}$, and 5 , respectively. Their coordinates are listed in the panels. The gray PKM profile in panel (c) magnified by a factor of 10 corresponds to the same position before the eruption. The sensitivity of the HK method is insufficient for this faint region. The densities and masses were computed for the sizes, $d$, specified in the panels, within the shaded temperature range.

partly by Ma et al. (2011). Having not detected the eruptive flux rope, the authors nevertheless established a sequential involvement of coronal loops in the motion from below upwards during the CME lift-off. Here we study the relation between the erupting flux rope and the CME formation.

\subsubsection{Rim and Inner Structures in $193 \AA$ AIA Images}

Figure 8 and the AIA_131_171_loops.mpg movie present some episodes of the CME genesis. The erupting flux rope visible in $131 \AA$ is shown in the left column. The red arc outlines its top according to the measurements in Figure 6. The arc is 
also superimposed on the $193 \AA$ images in the middle and right columns, where the flux rope is not visible. The middle column with the reduced-background $193 \AA$ images (Section 2.1) presents the pre-eruptive coronal arcade above the AR. Four individual loops 1-4 are approximately outlined by the color oval arcs. The right column shows a selection of $12 \mathrm{~s}$ running difference $193 \AA$ images. The loops appear in these images, when start moving.

The CME formation process presented in the right column of Figure 8 developed from below upwards, as the preceding studies concluded. This succession is confirmed by the progressively decreasing distances between loops $1-4$. The agent, which drove the loops, was an outward-propagating MHD disturbance. The distance between the red and dark-green arcs in the middle column (e.g., Figures [8: and 8]) also decreases; the flux rope (red) started to expand earlier and faster than the lowest loop 1 (dark-green). Thus, a probable driver of the expansion process forming the CME was the flux rope, which erupted at a very small altitude (left column).

The disturbance produced by the impulsively erupting flux rope is represented by the yellow circle. After the passage of this disturbance through loops 1 to 4 , they sequentially start moving (Figures $8 \mathrm{k}-8 \mathrm{~h}$ ). The loops become compressed to each other from below in Figures 8 and 80 .

The outward-propagating disturbance and the involvement of the loops in the expansion is demonstrated by the time history of the CME formation in onedimensional spatial profiles in Figure 9. The profiles were computed from the running-difference $193 \AA$ images in a fixed direction of $-36^{\circ}$ southward from the west and averaged over a 10-pixels wide slice. The image in Figure 9 is similar to the slit images presented by Ma et al. (2011) in their Figure 3 but shows more details due to a harder image processing.

Any moving feature appears here as an inclined strip, whose instant slope is its velocity. The traces of the four loops 1-4 shown in Figure 8 are clearly visible. Initially the loops rise slowly; in an interval of 05:35:40-05:37:30 their velocities considerably increase, remaining nearly constant afterwards. Note that the running differences only show the leading edge, while the trailing part disappears.

The red curve is the flux-rope plot from Figure 6 b. A faint trace is detectable $(15-20)^{\prime \prime}$ below it up to 05:38:30 and, possibly, later. This trace seems to belong to the flux rope, while the lag is partly due to the varying direction of its fastest expansion, mostly different from $-36^{\circ}$ (see Figures $5 \mathrm{5}-5 \mathrm{k}$ ). The presence of this trace in $193 \AA$ (and $131 \AA$ ) without any manifestations in 171 or $211 \AA$ indicates a temperature around $17 \mathrm{MK}$ in the detected part of the flux rope.

A fastest faint trace is detectable in the nearly radial direction after 05:36:10 even inside the forming $\mathrm{CME}$, starting at a height of $\approx 95 \mathrm{Mm}$. Its yellow outline was calculated for the wave propagation using Equation (11) in Section 2.2 with a wave onset time estimated for the flux rope's acceleration peak, $t_{0}=05: 35: 10$. A density falloff exponent, $\delta=2.5$, was adjusted to reach a best fit of the wave trace. The kinematical identity of the wave traces inside the rim and outside it rules out the bow-shock excitation by the rim. The yellow ovals in Figure 8 also correspond to this fit. Although the power-law fit was derived for a blast wave, the wave inside the forming CME, most likely, had not yet steepened into the shock (to be discussed in Section 7.1). 


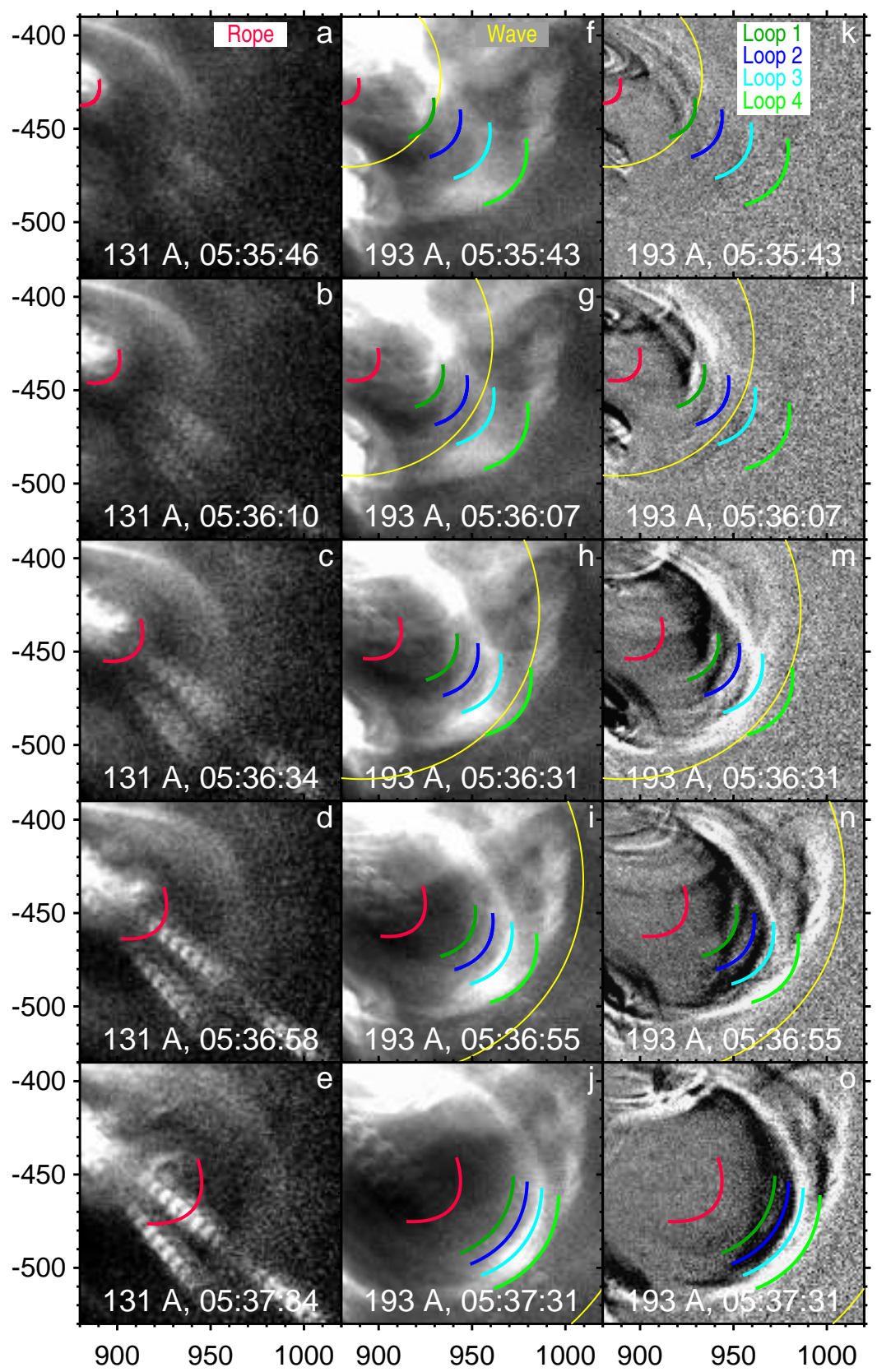

Figure 8. The eruptive flux rope in AIA $131 \AA$ images (left column, a-e) and the loops sequentially involved in the eruption observed in $193 \AA$ (middle and right columns). The middle column $(\mathrm{f}-\mathrm{j})$ presents the $193 \AA$ images with a reduced background. The right column $(\mathrm{k}-\mathrm{o})$ presents their 12-s running differences. The red arc outlines the top of the eruptive flux rope. The yellow arc outlines the wave. The four other color arcs approximately outline four conspicuous loops 1-4 of a pre-eruption arcade. 


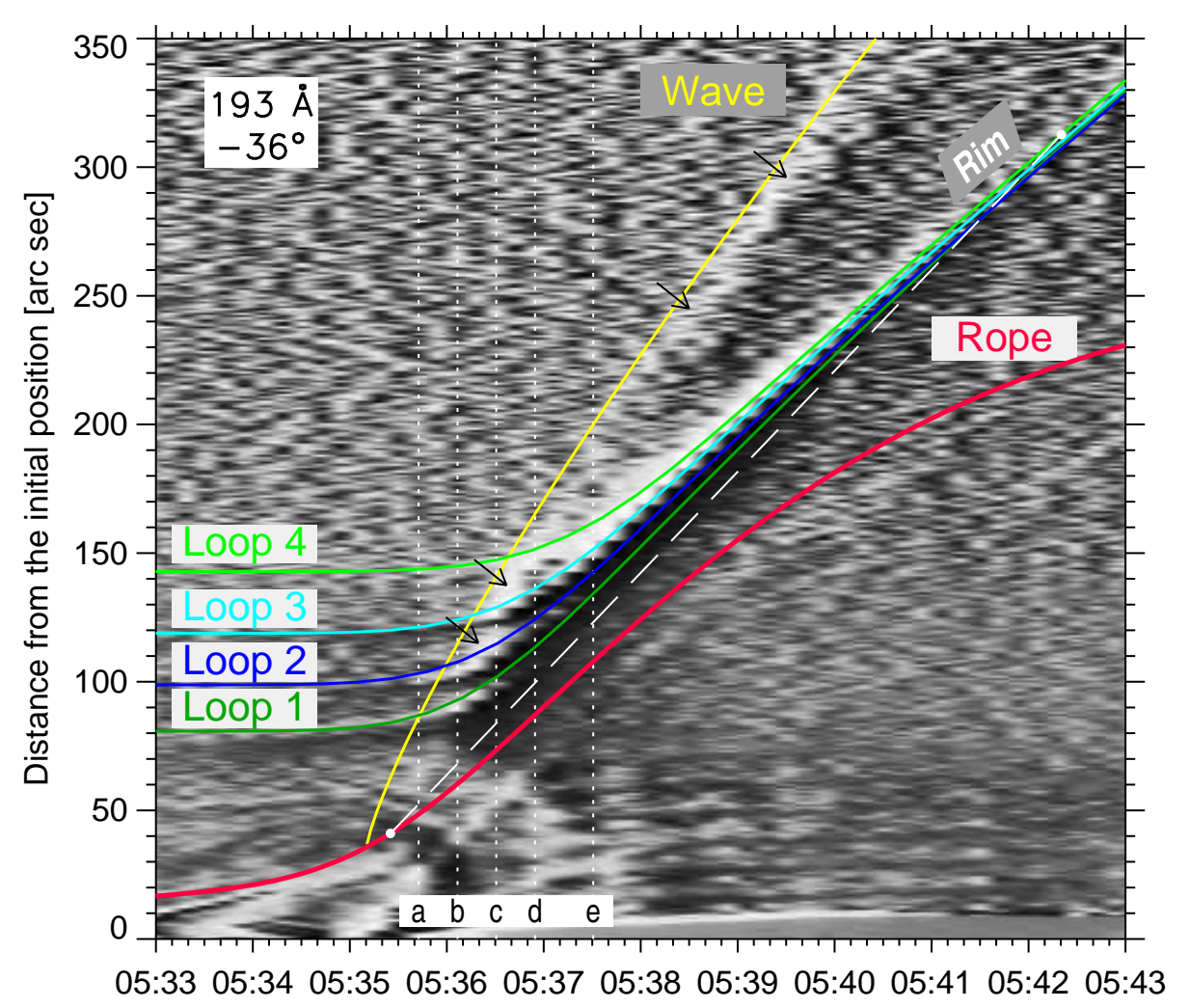

Figure 9. CME formation in one-dimensional spatial profiles computed from running-difference $193 \AA$ images in a direction of $-36^{\circ}$ southward from the west. The red curve represents the flux rope. The yellow curve outlines the trace of the wave. Some of its signatures are indicated by the arrows. The remaining color curves outline the trajectories of the expanding arcade loops (same colors as in Figure 8). The vertical lines mark the observation times of Figures $8 \mathrm{a}$. The labels of the corresponding panels are indicated at the bottom. The tilted dashed line denotes the trajectory of a virtual piston discussed in Section 7.1

The kinematics of loops 1-4 in Figure 9 can be inferred, keeping in mind that their expansion was limited from above by the rim. Initially the loops were static. The outward-propagating wave reached loops 1, 2, 3, and 4 one after another, and sequentially drove their expansion. Loop 1 acquired a highest speed and then had to decelerate, being restricted by the rim. The highest speeds, accelerations, and decelerations of loops 2,3 , and 4 slightly decreased one after another. The final speeds of the four loops converged to the final speed of the rim.

Four color curves outlining the trajectories of loops 1-4 were calculated analytically with parameters adjusted to match the traces of the loops. Eselevich and Eselevich] (2013) presented approximate height-time plots of the rising loops and proposed a fast emergence of a magnetic tube from below the photosphere at about 05:33. This idea contradicts the slow rise of the CME-progenitor coronal structures during the long-lasting pre-eruption heating and the early onset, by 05:31, of a sharp increase in the $131 \AA$ and 0.5-4 $\AA$ emissions in Figures 5 a - 5 b and Figure 6 . 


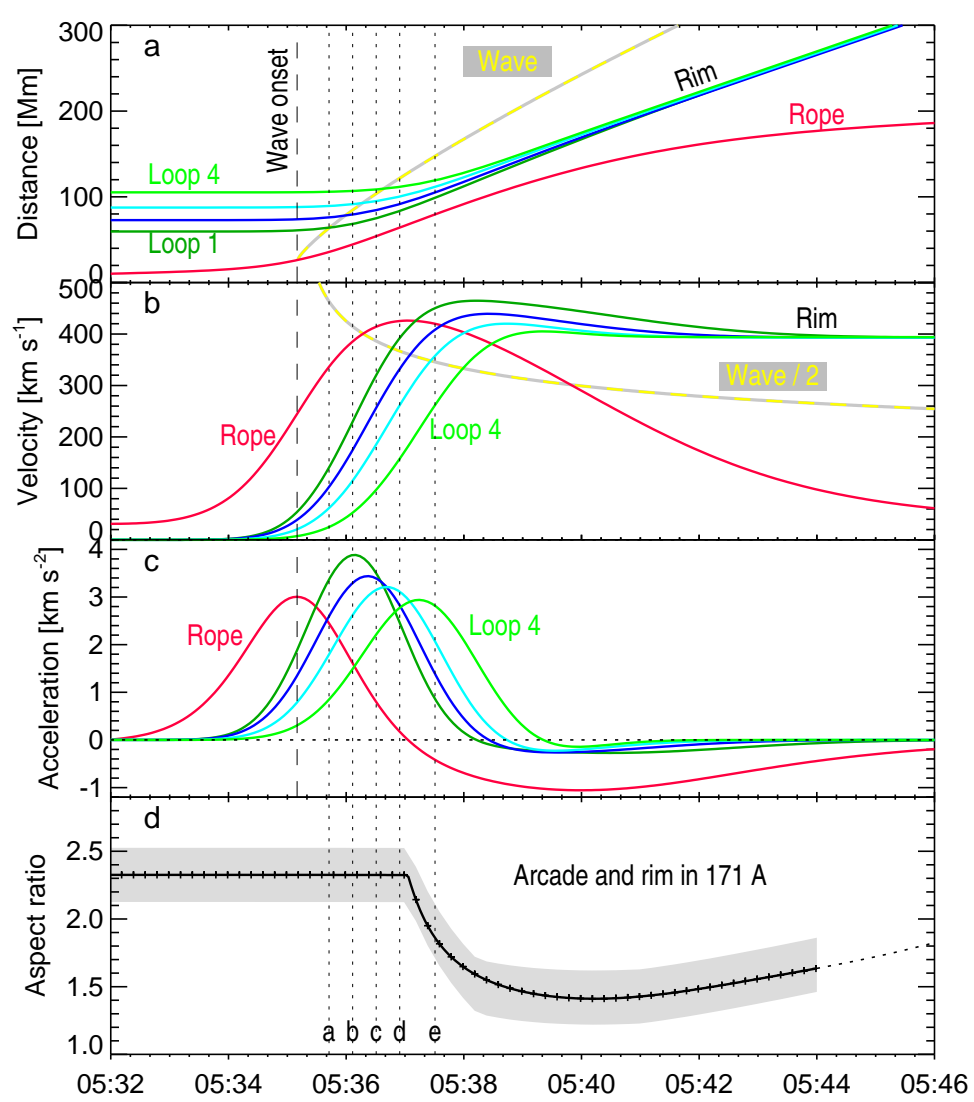

Figure 10. Kinematics of the flux rope, arcade loops $1-4$, and the wave (same colors as in Figures 8 and 9). (a) Distance-time plots similar to Figure 9 The vertical dotted lines mark the observation times of Figures $8 \mathrm{a}-8$. The labels of the corresponding panels are indicated at the bottom. The vertical dashed line marks the wave onset time. (b) Velocity-time plots. The wave velocity (dashed yellow-gray) in the plot is reduced by a factor of 2. (c) Accelerations of the flux rope (red) and arcade loops 1 - 4. (d) The measured aspect ratio of the arcade loop 4 and rim. The shading represents the uncertainty. The dotted extension approximately corresponds to the later expansion, when the rim is not reliably detectable.

Nevertheless, Eselevich and Eselevich (2013) correctly showed in their Figure 7a the sequential involvement of the loops in the motion.

The analytic color height-time plots in Figure 9 allowed us to obtain quantitative kinematics of the loops. The corresponding plots for the four loops, flux rope (red) and wave (dashed yellow-gray) are presented in Figures 10a 10.

Figure 10a reproduces Figure 9 without the background image. Figure 10p shows the velocity-time plots. The highest velocities reached by the flux rope and the four loops were between 400 and $500 \mathrm{~km} \mathrm{~s}^{-1}$. The wave speed was actually twice higher than the dashed yellow-gray curve shows, and started from $\gtrsim 1000 \mathrm{~km} \mathrm{~s}^{-1}$, which is a typical fast-mode speed in the low corona above an active region. Then the wave speed monotonically decreased all the time. 
The flux rope speed was $\approx 250 \mathrm{~km} \mathrm{~s}^{-1}$ at the wave onset time, $t_{0}=05: 35: 10$, and rose farther. Loop 4 and the forming rim started to expand well after the wave onset time, and therefore could not excite the wave. The relation between the velocities of the flux rope and wave rules out its bow-shock regime. Note that the loops accelerated gradually which indicates that the wave was not yet in the shock regime until, at least, its passage through loop 4 at 05:36:30 in the direction of the measurements $-36^{\circ}$ southward from the west. Otherwise, the velocity of a loop pushed by a shock wave should change abruptly.

Figure 10, presents the accelerations of the flux rope and loops. All components of the forming CME were obviously driven by the erupting flux rope, whose acceleration pulse led all others. Loops $1-4$ sequentially accelerated $\left(3-4 \mathrm{~km} \mathrm{~s}^{-2}\right)$ up to $400-500 \mathrm{~km} \mathrm{~s}^{-1}$, and then somewhat decelerated, approaching the final speed of the rim. A trivial deceleration of the wave is not shown.

Figure 10] shows the aspect ratio estimated for the top of the forming CME in manual outlining the curvature of loop 4, which then joined the rim (the black arc in Figure 3). The uncertainty is shown by the shading. We measured the aspect ratio by outlining loop 4 with an ellipse, whose lower edge was fixed at the photosphere, and did not endeavor to catch the whole shape of the loop, which was more complex. The aspect ratio variations are best visible in the AIA_131_171_loops.mpg movie, where loop 4 and the rim are outlined by the green arc. Comparison of Figures [10d and 10. reveals a similarity between the variations in the aspect ratio and the flux-rope deceleration. However, the aspect ratio increased slower than the deceleration ceased ( $c f$. the shapes of the two curves after 05:40). Thus, the variations in the aspect ratio of the CME bubble were probably governed by the flux rope expanding inside it, while its reaction had a reasonable delay.

It is worth to compare our measurements with the results obtained previously. Patsourakos, Vourlidas, and Stenborg (2010) were the first who measured the speed, acceleration, and the aspect ratio of the rim (CME bubble). They found that its speed reached a maximum of $400 \mathrm{~km} \mathrm{~s}^{-1}$ at $05: 38$ and then decreased to $300 \mathrm{~km} \mathrm{~s}^{-1}$. The maximum acceleration of $2 \mathrm{~km} \mathrm{~s}^{-2}$ was found to occur slightly after $05: 36$, followed by a deceleration up to $-0.5 \mathrm{~km} \mathrm{~s}^{-2}$ around 05:39. With quite different measurement techniques used by us and the authors, both results appear to be close to each other with an acceptable accuracy.

The aspect ratio variations we measured are basically close to those found by Patsourakos, Vourlidas, and Stenborg (2010), with somewhat larger differences due to different ways of the measurements. Substantial is a recovery tendency of the aspect ratio after 05:40 which is also indicated by the measurements of Gopalswamy et al. (2012) in their Figure 3b. Possible causes of these variations are discussed in Section 7.2 .

The difficulties to detect the earliest signatures of the wave and to measure its kinematics have resulted in a scatter between the results of different authors. Kozarev et al. (2011) found the wave speed of $\approx 735 \mathrm{~km} \mathrm{~s}^{-1}$ at 05:37 and its subsequent deceleration, very close to our results. The estimates by Ma et al. (2011) show a certain deceleration after 05:40 from $600 \mathrm{~km} \mathrm{~s}^{-1}$ to about $500 \mathrm{~km} \mathrm{~s}^{-1}$ at 05:42-05:44, also mainly consistent with our results. 


\subsubsection{Rim and Pileup on its Top in $211 \AA$ AIA Images}

To study the details of the pileup formation on top of the rim shown in Figure4. we consider the $211 \AA$ AIA images in a way similar to the preceding section. Here we use a different direction, in which the loops inside the rim are indistinct, but the rim, pileup, and wave trace are clearly visible. Figure 117 presents a timehistory diagram computed from running differences of the $211 \AA$ images in a direction of $-22^{\circ}$ southward from the west.

The trace of the arcade top represented by loop 4, which joined the rim, is distinct by about 05:40, and later it becomes poorly visible. Nevertheless, comparison of its faint trace with the dashed continuation of the earlier trajectory measured from Figure 9 indicates that the rim starts decelerating.

A much faster wave trace is outlined by the yellow fit. The $211 \AA$ data reveal an additional, slower bright branch outlined by a pink curve. It goes nearly parallel to the trace of the rim, being $\approx 90^{\prime \prime}$ higher. The high trace corresponds to the white arc in Figure 4 outlining the outer edge of the pileup. To relate it to the white-light CME, we calculated the kinematics of the frontal structure using the self-similar approximation (Grechnev et al., 2014b) with the parameters adjusted to coordinate the AIA and SOHO/LASCO observations. The parameters of the pink outline are $V_{1}=380 \mathrm{~km} \mathrm{~s}^{-1}$ and $r_{1}=264 \mathrm{~km}\left(360^{\prime \prime}\right)$ at $t_{1}=05: 41: 00$, and $V_{\infty}=190 \mathrm{~km} \mathrm{~s}^{-1}$ (the solid line in Figure 14). The compression of the loops to the rim indicates that the self-similar approximation did not yet fully apply at 05:41:00 (otherwise, the CME would expand uniformly); nevertheless, the pink outlines in Figures 11] and 11] appear to be acceptable. Our technique does not yet allow smooth concatenating the green rim's velocity with the pink CME speed, although they are close to each other.

To understand the pileup formation better and to figure out the properties of the wave, we consider a similar diagram in Figure 12 computed in the same direction from fixed-base difference $211 \AA$ images. It was very difficult to reveal individual structures between the rim and the leading edge, and therefore their separate traces outlined by the black dashed lines are regrettably faint. Several attempts showed that the errors in estimating the slopes of the faint traces in Figure 12 a (i.e., their velocities) did not exceed $\pm 7 \%$.

Previous studies assumed that the shock formed before 05:37, when the type II burst started, but it is unknown when the wave entered the shock regime in the direction of $-22^{\circ}$ in Figure 12. One cannot recognize if the transition between the horizontal dashed lines left and right from the wave front was abrupt (shock) or gradual. For the Mach number, $M$, it is only possible to estimate the upper limit, $M_{\max }=V_{\text {sh }} / V_{\text {fast }}$, so that $1 \leq M \leq M_{\max }$.

With known velocities of the wave front, presumably shock, $V_{\mathrm{sh}}$, and a structure moved by the gas behind the shock, $U_{\text {sh }}$, one can estimate the fast-mode speed, $V_{\text {fast }}$, from an equation $V_{\mathrm{sh}} \approx V_{\text {fast }}+\kappa U_{\mathrm{sh}} / 2$; the $\kappa$ coefficient governs the wave steepening rate (Grechnev et al., 2011b). This coefficient, $1 / 2 \leq \kappa \leq 3 / 2$, depends on plasma beta and the propagation direction Afanasyev and Uralov, 2012, Figure 8). Most likely, here we are dealing with a wave propagation nearly perpendicular to the magnetic field in low-beta plasma, $\kappa \approx 3 / 2$. The estimates of the maximum Mach number and the fast-mode speed for four instants are 


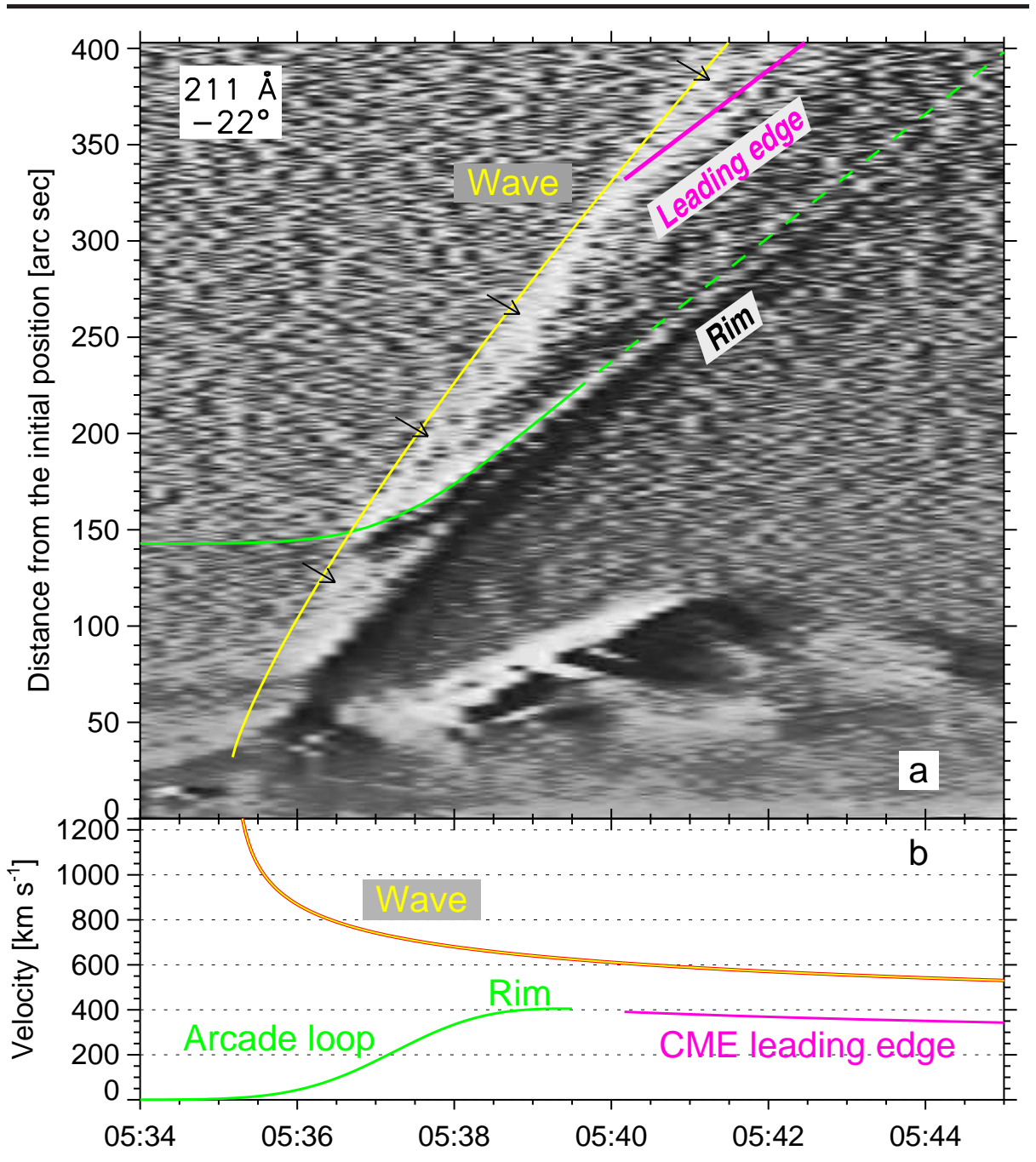

Figure 11. (a) Time history of the CME formation in one-dimensional spatial profiles computed from running-difference AIA $211 \AA$ images in a direction of $-22^{\circ}$ southward from the west. The yellow curve outlines the wave trace. Some of its signatures are indicated by the arrows. The green curve corresponds to the arcade top (loop 4) clinging to the rim. The later part of the rim signature shows a stronger deceleration then loop 4 in Figures 9 and 10 (the dashed continuation of the green curve) had. The outermost signature of the bubble is outlined with the pink curve corresponding to the leading edge of the CME frontal structure (the top of piled-up plasma). (b) Velocity-time plots for the wave (yellow on gray), the upper arcade loop 4 and the rim (green), and the CME leading edge (pink).

shown in Figure 12 b. Such estimations do not depend on the blast-wave or bow-shock regime, while their accuracy decreases for strong shocks.

Since the trajectory of loop 4 in Figure $12 \mathrm{a}$ is gradual, the discontinuity had not yet formed at 05:36:45. Figure 12] indicates that the Mach number could only increase from 05:37:25 to 05:40:00, not exceeding $M_{\max }$, which was nearly constant, $1.45 \leq M_{\max } \leq 1.65$. The wave probably evolved from a linear fast- 


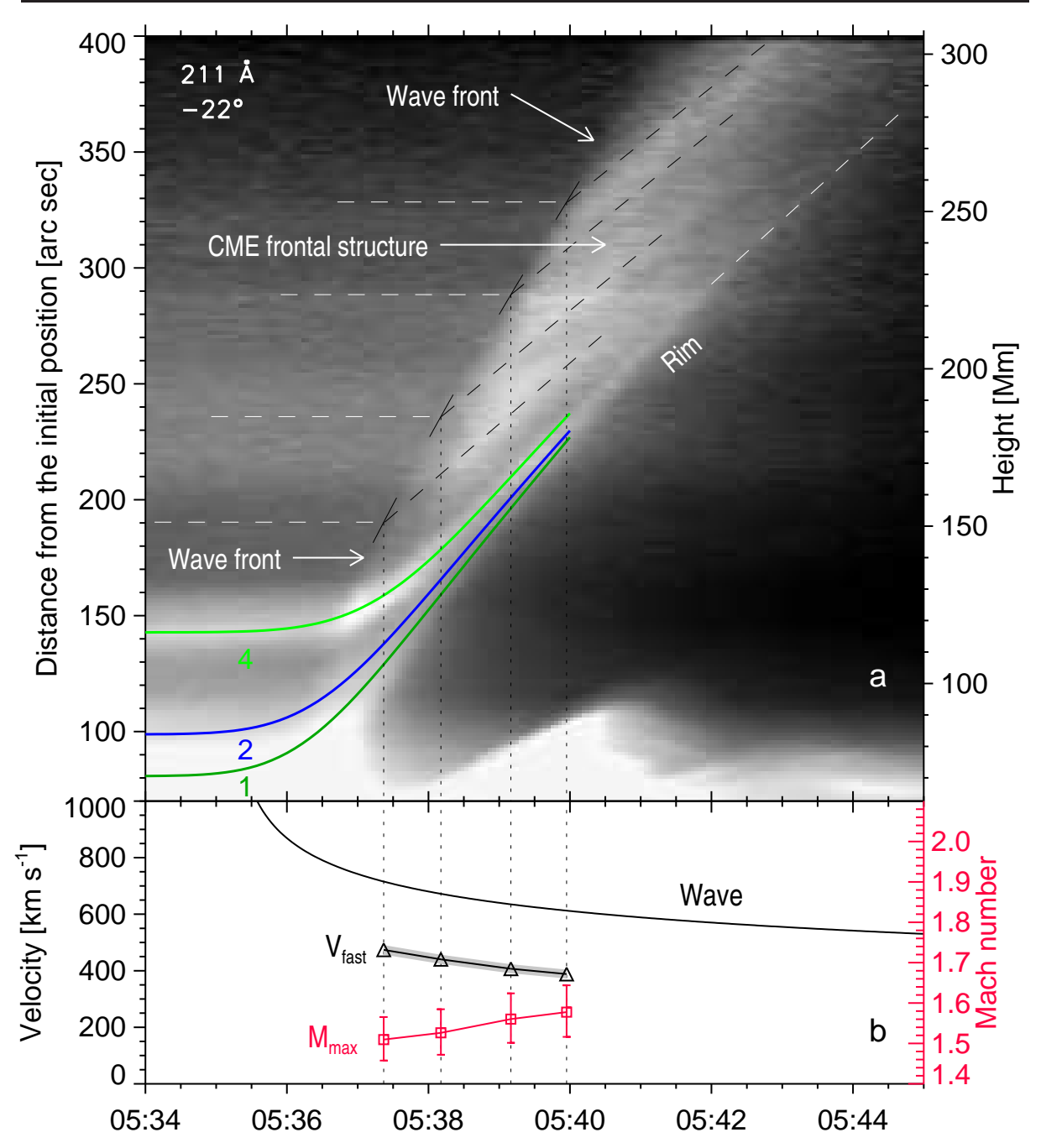

Figure 12. (a) Formation of the CME frontal structure in spatial profiles computed from $211 \AA$ fixed-base differences in a direction of $-22^{\circ}$ southward from the west. The black dashed lines outline individual structures involved in the motion by the wave, whose front is outlined by the rare dashes. The white horizontal dashed lines left of the wave front denote presumable initial positions of these structures. The color curves labeled 1, 2, and 4 correspond to the loops discussed previously. The inclined white dashed line outlines the rim. (b) Velocity-time plots for the wave (long black curve), the maximum Mach number (red squares) with uncertainties, and the calculated fast-mode speed (triangles) with uncertainties shown by the shading.

mode wave $(M=1)$ to a simple wave and then steepened in some time into the shock with $M<M_{\max }$. The shock might have not formed at all in this direction, right ahead of the CME. The source of the type II burst could be located at a flank of the wave front-e.g., in a streamer-like structure in Figure 4 .

The fast-mode speed shown in Figure $12 \mathrm{~b}$ was calculated under an assumption of the weak shock regime of the wave. If this was not the case for all the four instants, then the real fast-mode speed should be between the line connecting 


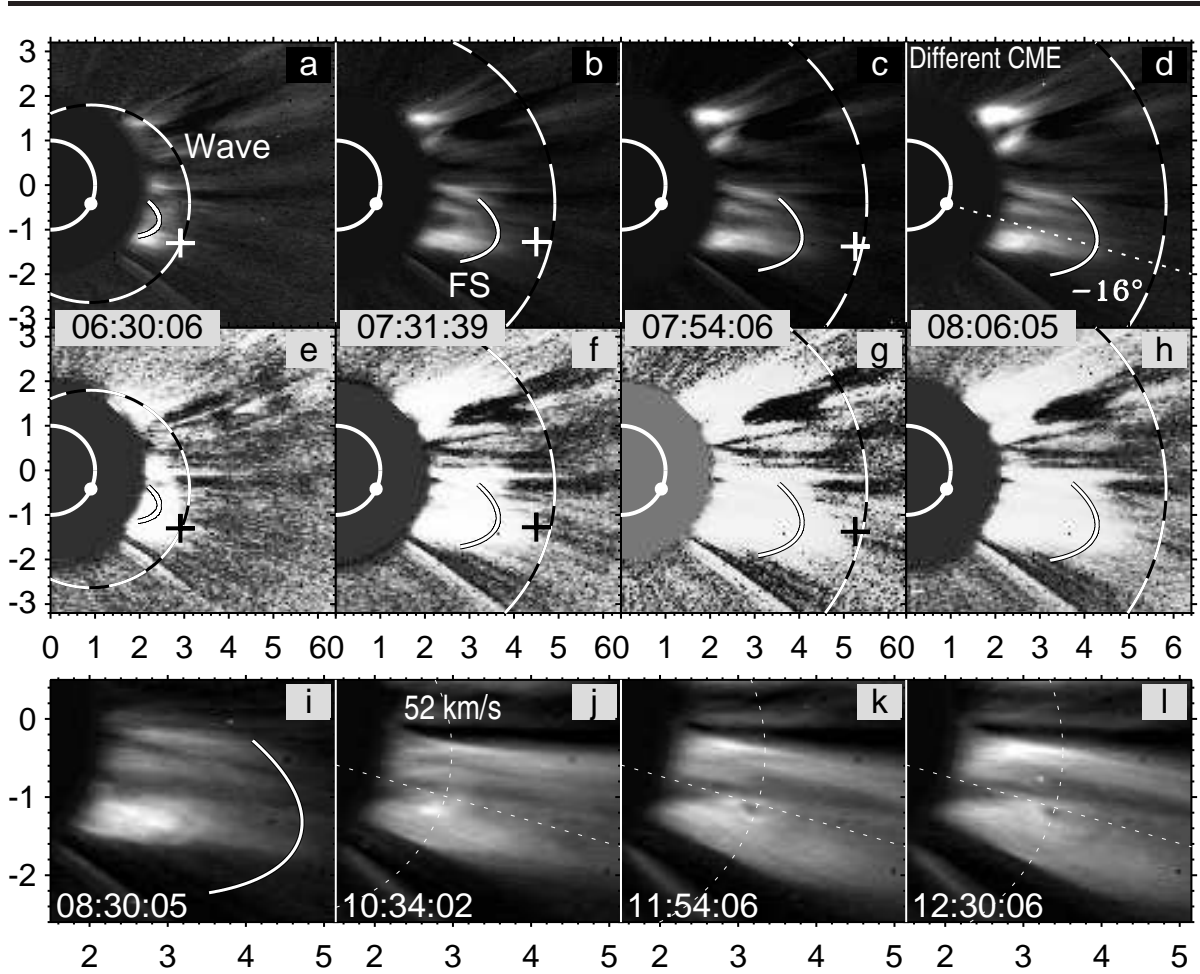

Figure 13. LASCO/C2 images: CME expansion (upper row, fixed ratios), wave traces (middle row, running differences), and the CME structure (lower row, fixed ratios). The small oval arcs outline the leading edge of the frontal structure (FS). The larger dashed circles in the upper and middle rows outline the wave traces. The crosses denote the measurements in the CME catalog. The dotted circle in the lower row corresponds to the velocity of the flux rope's center of $\approx 52 \mathrm{~km} \mathrm{~s}^{-1}$. The solid circle denotes the solar limb. The small filled circle denotes the eruption site. The axes present the coordinates from the solar disk center in solar radii.

the triangles and the higher plot of the wave, anyway decreasing with height. The decrease is typical of the fast-mode speed above active regions at heights $<0.4 R_{\odot}$ (Dulk and McLean, 1978, Gary, 2001, Mann et al., 2003).

In summary, Figures 11 and 12 demonstrate that CME formed due to the outward-propagating wave, which swept up all the structures in its way, involving them in the expansion. The CME frontal structure was mostly constituted by the pileup on top of the expanding rim that previously was a relatively high environment of the active region.

\subsection{CME Expansion Visible in White Light}

To validate our results drawn from the EUV observations of the eruption, it is important to coordinate them with a white-light CME observed by SOHO/LASCO. Figure 13 shows selected LASCO/C2 images. The CME leading edge and wave traces are outlined according to the kinematics presented in Figure 14.

The upper and middle rows of Figure 13 shows different representations of the same four images to reveal a poorly visible CME structure in the upper row 


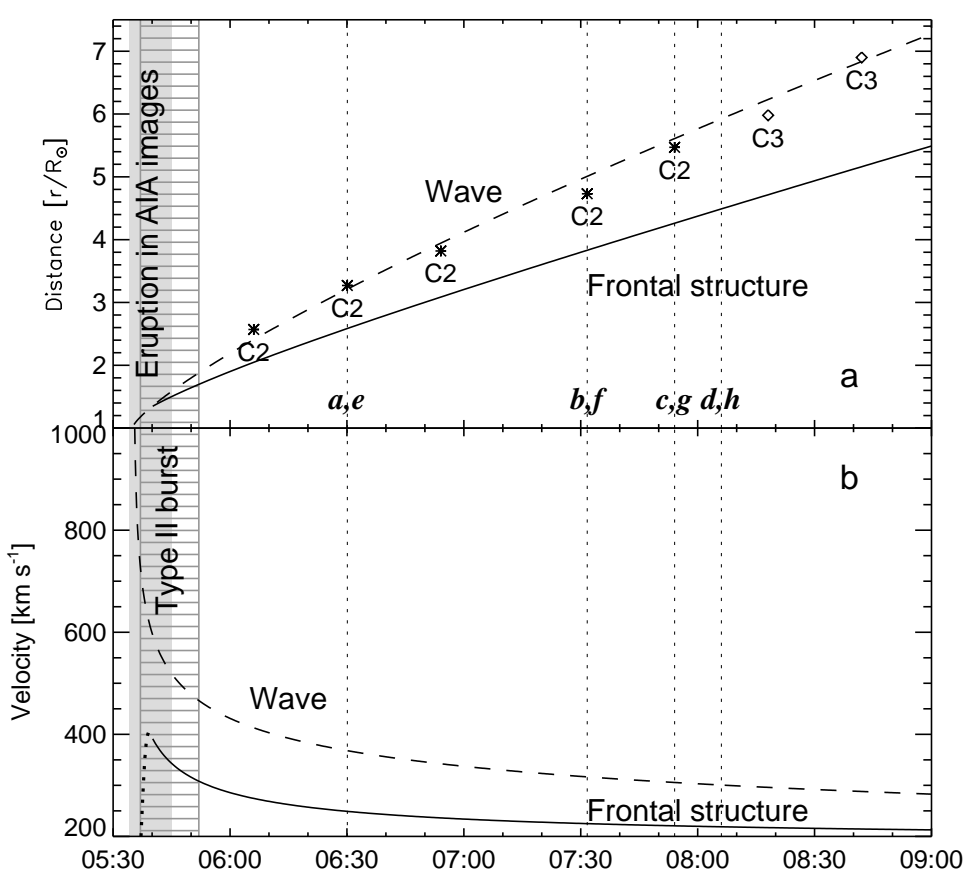

Figure 14. Heliocentric distance-time (a) and velocity-time (b) plots for the leading edge of the CME frontal structure (solid) and the wave (dashed). The symbols in panel (a) represent the measurements from the CME catalog. The vertical dotted lines denote the times of the images in Figure 13 The labels of the corresponding panels are indicated at the bottom of panel (a). The initial accelerating part (dotted) in panel (b) corresponds to the arcade loop and the rim in Figure 11 The gray shading corresponds to the time interval presented in Figure 11] The horizontal hatching denotes the interval, in which the type II burst was observed.

and to detect faint wave traces in the middle row. The faint frontal structure (FS) is outlined by the oval arc, whose increasing radius corresponds to the pink self-similar fit in Figure 11. It seems to consist of stretched loops. The rim is not pronounced in the CME structure. The CME orientation still turned from the initial $-46^{\circ}$ in Figure 5 f to $-16^{\circ}$ in Figure 13 d (position angle of $254^{\circ}$ in the CME catalog http://cdaw.gsfc.nasa.gov/CME」ist/; Yashiro et al., 2004).

The crosses in the upper and middle rows represent the measurements in the CME catalog. They were made for the fastest feature, being close to the wave traces outlined by the dashed circle. The wave outline is the same as we used in the preceding section, with $t_{0}=05: 35: 10$ and $\delta=2.5$.

The four later $\mathrm{C} 2$ images in the lower row reveal a flux-rope structure of the CME core. The average speed of its center marked with the dotted circle in Figures 13-13. is $52 \mathrm{~km} \mathrm{~s}^{-1}$, consistent with our measurements in Figure 6. The flux rope, whose initial expansion drove the whole CME formation process, later relaxed and became the CME core visible well behind the leading edge.

The kinematic evolution of the FS and the wave ahead it is clear from Figure 14. The FS was probably formed from coronal loops swept up by the expanding rim, whose velocity is plotted in Figure 14 $\mathrm{b}$ with the dotted line. Being expelled by the erupting flux rope, the wave initially was fast and possibly 
strong enough to produce the type II emission within the hatched interval. The wave speed in the radial direction decreased within this interval from $\approx 680$ to $\approx 460 \mathrm{~km} \mathrm{~s}^{-1}$. Note that the type II burst source could be located in a different direction, where the wave strength might be also different.

Then the wave strongly decelerated and dampened, being not driven by the trailing piston, which considerably slowed down. The evolution of the wave speed inferred from AIA and LASCO observations does not confirm an assumption of some authors about its possible peak between $1.5 R_{\odot}$ and $2.6 R_{\odot}$ (the appearance in the LASCO field of view). Although the wave and CME were kinematically similar, the wave speed at distances $>2 R_{\odot}$ was too low for the bow-shock regime. Then the wave speed still decreased below $300 \mathrm{~km} \mathrm{~s}^{-1}$ at about $7 R_{\odot}$, comparable to the solar wind speed, that points to its decay into a weak disturbance. The increasing role of the solar wind is confirmed by a subsequent acceleration of the CME suggested by the measurements in the CME catalog.

\section{Wave Signatures and EUV Transient}

\subsection{EUV Wave}

Most preceding studies considered a transient expanding in EUV images (EUV wave; Warmuth, 2015) as a signature of a shock wave, assuming its bow-shock regime. We showed in the preceding sections that the EUV wave moving away from the Sun consisted of swept-up plasmas on top of the expanding separatrix surface, with which the rim was associated. The pileup was involved in the motion by the outward-propagating wave, which was initially excited by the impulsive expansion of the flux rope, and then resembled decelerating blast wave.

The propagation conditions along the solar surface are considerably different from those away from the Sun. In the lateral directions, the separatrix surface does not follow the expanding wave up to large distances. It is therefore important to study the EUV wave propagating in different directions.

Downs et al. (2012) analyzed the EUV wave in this event in realistic coronal conditions on the basis of a thermodynamic MHD simulation. They stated a clear distinction between the wave and non-wave component and concluded that the propagating EUV transient exhibited the behavior of a fast-mode wave. However, it was difficult to ascertain the wave excitation scenario in this simulation.

Conversely, our approach only allows studying global properties of a wave, without a reference to realistic inhomogeneous corona. We will nevertheless try to reconcile the wave excitation scenario revealed with a posterior near-surface wave propagation and examine how acceptable its fit in this case works.

The EUV wave was observed from two vantage points by SDO/AIA and STEREO-A. Some EUVI $195 \AA$ images are shown in Figure 15] where their nominal observation times are specified. The kinematical plots corresponding to the ellipses outlining the EUV wave are presented in Figure 16, where the EUVI observation times are referred to the SDO vantage point. The wave kinematics along the spherical solar surface was calculated with the same onset time as previously, $t_{0}=05: 35: 10$, and a density falloff exponent of $\delta=2.1$. The blue 


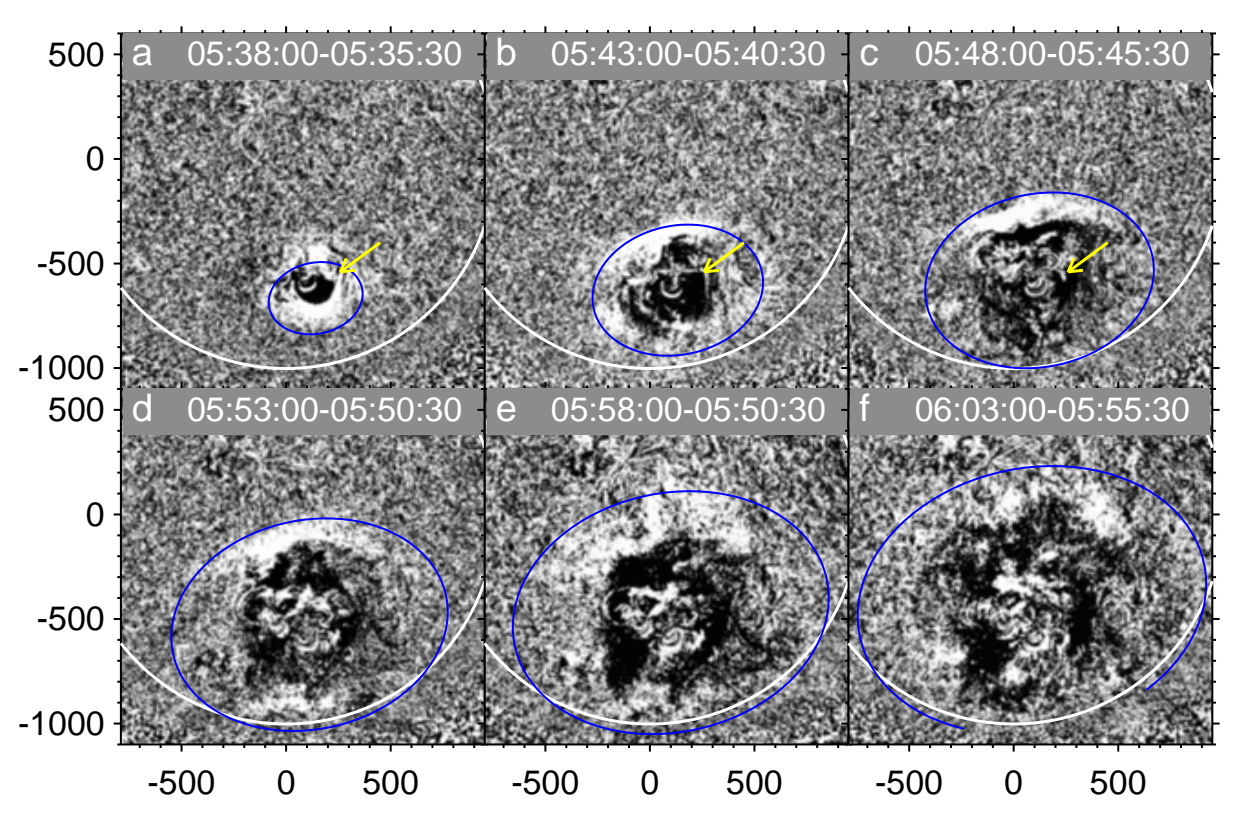

Figure 15. EUV wave propagation in STEREO-A/EUVI $195 \AA$ running difference images. The blue ellipses represent calculated wave fronts. The yellow arrow in panels (a)-(c) points at a base of the streamer-like feature (denoted in Figure 4 ), in which the source of the type II burst could be located. The white circles outline the solar limb. The axes show the coordinates in arcsec from the solar disk center.

ellipses in Figure 15 (yellow in the EUVI_wave.mpg movie) delineate the nearsurface isotropic trail of an expanding global wave front. This trail corresponds to an effective height of $35 \mathrm{Mm}$ and the wave propagation in the corona without any inhomogeneities. The ellipses were calculated as small circles at a sphere with a pole coinciding to the eruption center.

The ellipses tolerably correspond to the leading edge of the bright EUV wave in the images, although the initial wave could actually be somewhat faster than the outline. The effective height might not be constant, and a small displacement of the wave 'epicenter' is not excluded. Such a displacement toward a region of a higher fast-mode speed was reported previously (see, e.g., Grechnev et al., 2013a). The faintness of the EUV wave here disfavors detection of this effect.

The most probable source of a type II radio burst is the current sheet of a small coronal streamer stressed by a shock front (see Section 6.2). The type II burst in this event started at 05:37:00 (Figure 19). The yellow arrow in the upper row of Figure 15 points at the base of a streamer-like feature visible in the AIA $211 \AA$ image in Figure 4 . The wave front in Figure 15 has already passed this feature, while the observation time of this STEREO-A/EUVI image corresponds to $05: 37: 31$ UT. Thus, the actual positions of the wave front do not contradict a possible location of the type II burst source in this feature.

Figure 17 presents the EUV wave propagation as seen by AIA in 211 and $171 \AA$. The off-limb front is outlined by the blue curves composed from three oval arcs adjusted to fit the wave kinematics measured from the quadrature 


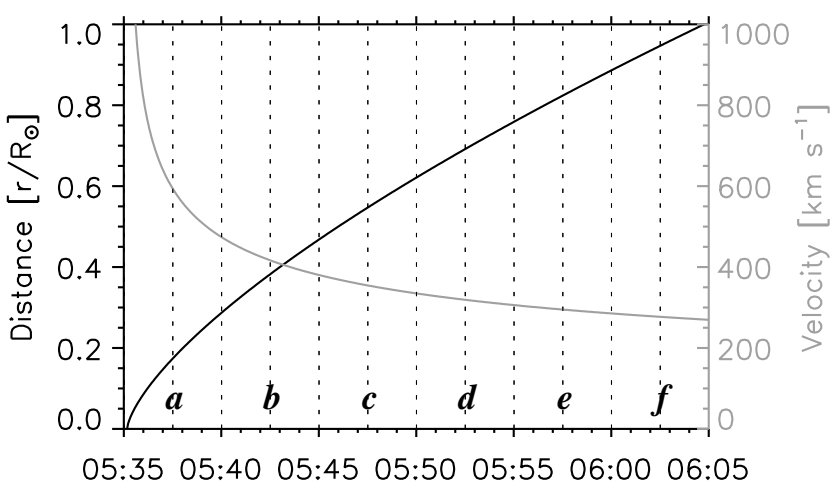

Figure 16. Distance-time (black) and velocity-time (gray) plots of the EUV wave propagation from the eruption center along the solar surface. The vertical lines mark the observation times of Figures [15] - 15] corrected for the difference between the orbits of the Earth and STEREO-A, as if the Sun were viewed from SDO. The labels of the corresponding panels are indicated at the bottom.

observations with STEREO-A/EUVI and SDO/AIA. The orientations of the north and south arcs were progressively adjusted to catch the tilt of the wave front that apparently varied in its motion along the limb. The radius of the south arc calculated from the power-law fit was stretched by a constant factor to catch a faster wave propagation toward the south pole, where the fast-mode speed was higher in the region of the polar coronal hole. The green ellipses were calculated for the surface trail of the wave front visible on the Earth-facing hemisphere.

The EUV transient appears between the calculated wave front and the rim as a brightening in the higher-temperature $211 \AA$ images and as a darkening in the lower-temperature $171 \AA$ images. The brightening in the first $171 \AA$ image in Figure 17, is due to separate loops, which have not yet merged into the thin rim. The EUV transient is most likely due to the pileup, while the different appearance in the two different-temperature channels indicates its heating.

Figure 18 compares the appearance of the EUV transient in the $193 \AA$ and $211 \AA$ images. They show a large difference between the orientations of the rim (along with the arcade loops pressed to the rim) and the long loops above it. This fact corroborates the association of the rim with a separatrix surface.

Comparison of Figures [18 and [18 indicates a larger opacity of the pileup on top of the rim in the higher-temperature $211 \AA$ channel (excluding the hottest window in $193 \AA$ ) and some temperature increase from the outer edge of the pileup to the rim. The appearance of the pileup suggests that it was a thick, nearly spherical layer, bounded by the rim from inside, as shown in Figure 18.

In summary, the EUV transient enveloping the rim was the pileup and became the CME frontal structure. The near-surface EUV transient observed at large distances in Figures 15b-15f and moving toward the south pole in Figures 17b177 was a trace of the wave, which was not followed by CME structures. Having risen and expanded enough, the CME was not detectable from STEREO-A against the solar surface due to a strong decrease of its emission measure. 


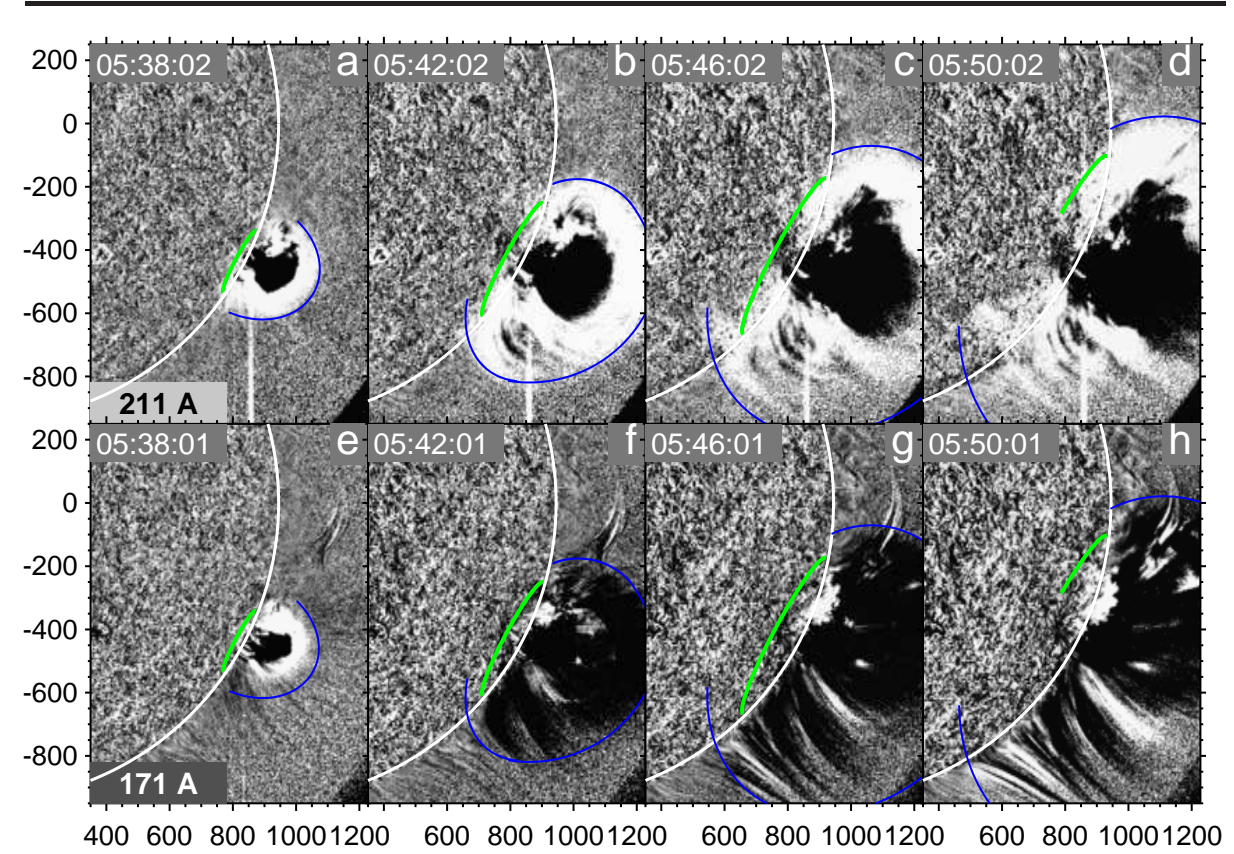

Figure 17. EUV wave propagation in AIA 211 and $171 \AA$ fixed-base image ratios to those at about 05:34:00. The blue curves outline the off-limb wave front. The green ellipses outline its surface trail. Two right panels reveal the wave reflection in the southern region. The white circles trace the limb. The coordinates are in arcsec from the solar disk center.

\subsection{Type II Burst}

The type II burst in this event was analyzed previously (Kozarev et al., 2011; Ma et al., 2011: Gopalswamy et al., 2012, Vasanth et al., 2014: Kouloumvakos et al., 2014) based on data from different radio spectrographs, each of which has its own ad-
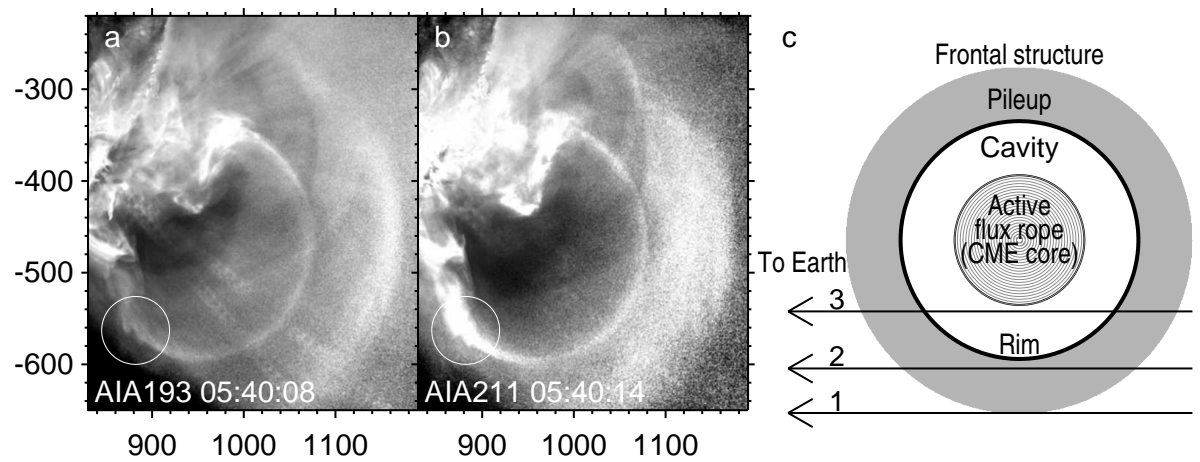

Figure 18. CME bubble in the $193 \AA$ (a) and $211 \AA$ (b) background-subtracted images at the indicated times divided by the images averaged within 05:31:45-05:33:25. The circle outlines the trace of a lateral disturbance, which follows the flux-rope liftoff and runs along the separatrix. The sketch in panel (c) presents the bubble as viewed approximately from STEREO-A and illustrates the brightness distribution visible from SDO. 


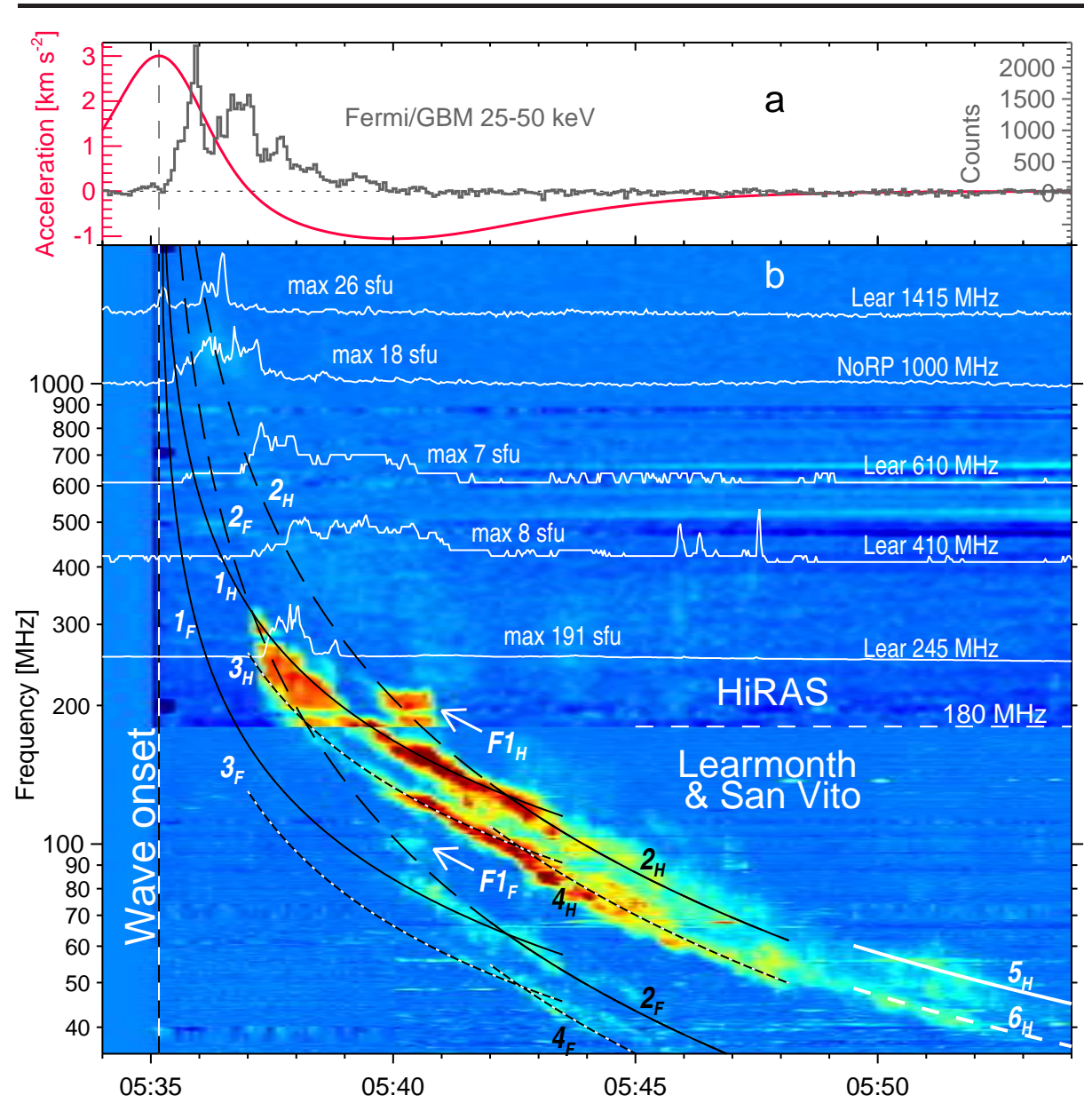

Figure 19. Relations between the eruption, HXR burst, and radio signatures of the shock wave. (a) Acceleration of the flux rope (red) and the HXR burst (gray). The vertical dashed line marks the wave onset time. (b) Dynamic spectrum of the type II burst composed from the HiRAS data $(>180 \mathrm{MHz})$ and Learmonth and San Vito RSTN data $(<180 \mathrm{MHz})$ along with normalized fixed-frequency time profiles (Learmonth and NoRP). The harmonically related pairs of the type II bands are outlined by the calculated trajectories $\left(1_{\mathrm{F}}, 1_{\mathrm{H}}\right),\left(2_{\mathrm{F}}, 2_{\mathrm{H}}\right)$, etc. of different line styles. An additional harmonic feature $\mathrm{F} 1_{\mathrm{F}}, \mathrm{F} 1_{\mathrm{H}}$ is indicated by the arrows.

vantages and limitations. We have combined a wide-range HiRAS spectrum with higher-resolution spectra recorded at the Learmonth and San Vito USAF RSTN stations to enhance their quality. Figure 19p presents the combined spectrum along with higher-sensitivity fixed-frequency records from the Learmonth RSTN radiometers and Nobeyama Radio Polarimeters (NoRP; Torii et al., 1979) at 1 $\mathrm{GHz}$. Their pre-burst levels correspond to their frequencies, and the peak fluxes are specified just after the bursts. The bursts correspond to a faint drifting continuum suggested by the HiRAS spectrogram. For comparison Figure 19a shows the acceleration of the flux rope and the HXR burst (similar to Figure 6 d). The vertical dashed line denotes the wave onset time, $t_{0}=05: 35: 10$. 
Dynamic spectra generally present superposition of emissions, which originate at different sites. The combined spectrum reveals a complex multi-lane structure of the type II burst that is barely visible in individual spectrograms. To understand this structure, we outline the trajectories of separate lanes with the curves of different line styles and colors. The ' $\mathrm{F}$ ' subscripts denote the fundamental and ' $\mathrm{H}$ ' the harmonic emission. All of the curves correspond to a single shock with an onset time $t_{0}$, crossing various coronal structures located in different directions from the wave origin. The different curvatures of the trajectories are most likely due to different plasma density falloffs, $\delta$, in the corresponding directions.

The fixed-frequency data reveal the early wave signatures before the onset of the type II burst. The outlining curves cross them at the onset or rise of the bursts at fixed frequencies, if the radiometers were sensitive enough to detect their fluxes, all of which did not exceed 30 sfu, being $<10$ sfu below $700 \mathrm{MHz}$. The type II burst started at 05:37:00, when the wave front has already passed through the rim and expanded farther away. The harmonic type II emission was much stronger than the preceding drifting continuum and reached $191 \mathrm{sfu}$.

The initial trajectories of the paired type II bands 1 and 3 are outlined with density falloff exponents of $\delta_{1}=\delta_{3}=2.05$, which are close to $\delta=2.1$ found in Section 6 for the propagation of a spherical wave along the solar surface. The fundamental emission is visible only occasionally. Then the bands had turns, which the outlining curves emphasize. At 05:43:00, bands 1 and 3 passed into bands 2 and 4 , respectively $\left(\delta_{2}=\delta_{4}=2.58\right)$. Another turn occurred between 05:48:00 and 05:49:30 from harmonic bands $2_{\mathrm{H}}$ and $4_{\mathrm{H}}$ to bands $5_{\mathrm{H}}$ and $6_{\mathrm{H}}$, respectively $\left(\delta_{5}=\delta_{6}=2.75\right)$. The corresponding fundamental bands left the observed frequency range. The bands before and after the turns overlapped.

The $\delta=2.58$ is not much different from $\delta=2.5$ found in Section 5 for the wave propagation away from the Sun, being close to the equatorial Saito model (Saito, 1970, see also Grechnev et al., 2011b). A nearly radial direction is also appropriate for $\delta=2.75$, corresponding to the Saito model at a higher latitude.

These facts suggest that the type II emission originated initially at a lowerlatitude flank of the wave front, and then, possibly, at both flanks, while the sources moved away from the Sun. A number of studies converge to the idea that a probable source of a type II burst is the current sheet of a small coronal streamer stressed by a shock front. This causes a flare-like process running along the streamer (e.g., Uralova and Uralov, 1994, Reiner et al., 2003, Mancuso and Raymond, 2004). A large-scale shock front crossing a wide range of plasma densities in the corona can only produce a drifting continuum (Knock and Cairns, 2005); the narrow-band harmonic emission can originate in a distinct extended narrow structure like a coronal ray. This scenario accounted for some structural features of type II bursts observed in different events (Grechnev et al., 2011b, 2011a, 2014b, 2015). Imaging meter-wave observations of type II sources support this scenario (Feng et al., 2013; Chen et al., 2014, Du et al., 2014).

The split bands $1-2-5$ and $3-4-6$, which are better visible in the harmonic emission $(\mathrm{H})$ in Figure 19, have been interpreted in terms of the emissions upstream and downstream of the shock front (Smerd, Sheridan, and Stewart, 1974). The dynamic spectrum shows an additional harmonically related equidistant feature $\left(\mathrm{F} 1_{\mathrm{F}}, \mathrm{F} 1_{\mathrm{H}}\right)$, which resembles the split bands in the slope at that time. 
However, the traditional interpretation cannot account for a third paired band. Du et al. (2014) presented observations of type II bursts also challenging to this interpretation. Grechnev et al. (2011b, 2015) proposed that the band-split type II bursts could be due to emissions from two nearby streamers. The complex structure of the type II burst in this event could be due to both scenarios. The Smerd, Sheridan, and Stewart (1974) scenario might correspond to the paired bands $1-2-5$ and $3-4-6$, and not to the $\left(\mathrm{F} 1_{\mathrm{F}}, \mathrm{F} 1_{\mathrm{H}}\right)$ feature. Note that, at least, one streamer is necessary in either scenario to get a narrow-band emission, which is crucial to make the band-splitting detectable.

The results of this section quantitatively agree with the wave development revealed in Sections 5 and 6 and show that some of the considerations and conclusions of the preceding studies need refinement. Relating the type II emission source to the region ahead of the CME nose, invoking the bow-shock properties for the estimations of coronal parameters on the way of the wave front, the assumption of its cylindrical geometry, and some others are among them.

\section{Discussion}

The 13 June 2010 event presents a rare case when the kinematical measurements of the features detectable inside the developing CME allows one to figure out the formation of its structure. The initiator of the event was a small eruptive filament. The outcome of the eruption was the appearance of an expanding coronal wave, whose front resembled a semi-sphere, with a spheroidal cavity inside bounded by the rim. From the observational point of view, this event was strikingly similar to the SOL1996-12-23 event addressed by Dere et al. (1997), who demonstrated for the first time the development of a large-scale CME from a small volume. However, the active role in our event of the eruptive filament located inside the cavity was underestimated.

This view was probably the major reason to interpret this event in terms of a popular concept, which assigns to the coronal cavity a key role in the coronal wave excitation and creation of the CME itself. This concept considers the cavity as a cross-section of a large magnetic flux rope, a major driver of a CME. The filament (prominence) is regarded as a passive element embedded in the flux-rope structure. The filament eruption is considered, at most, as a trigger destabilizing the large flux rope. This view is popular, although filaments erupting from active regions resemble small flux ropes, and their behavior does not depend on their large-scale environment. The filaments expand during the acceleration stage earlier and sharper than other structures; their shapes can rapidly change, according to their small sizes. The impression of the dominant role of a large flux rope, most likely illusive, probably appeared due to relatively slow eruptions of extended filaments outside of active regions, as discussed in Grechnev et al. (2015, Section 4.1).

The traditional concept of a flux rope rooted in the photosphere only by two of its ends simplifies the magnetic structure of observed filaments. A real filament has additional lateral connections to the photosphere by numerous threads. It is not clear what is the surface of the magnetic rope related to a real filament. 
This surface cannot exceed the magnetic domain containing the pre-eruption filament. Most likely, the cavity in the 13 June 2010 event was bounded by the magnetic domain containing the pre-eruptive filament, because its photospheric base was fixed, being confined by the separatrix surfaces.

In the traditional view, the only source of a coronal wave is the outer surface of the large flux rope, which acts as an expanding piston. The rim in our event looks very similar to this surface that led to their unfounded identification in previous studies. A popular assumption was also postulated that the wave can only become a shock, if the cavity (rim) expands with a super-Alfvénic speed. However, this bow-shock scenario is not the only possible mechanism of the shock-wave excitation (Vršnak and Cliver, 2008).

\subsection{What Was the Driver of the CME and Coronal Wave?}

There are two options: either a large flux rope, which occupies the whole volume of the cavity, or a small flux rope formed from the erupting filament inside the cavity. These two options seem to be very similar. A basic question related to the CME formation is what drove the wave at different stages of the event. In other words, we ask if the surface of the piston was inside the cavity or at its boundary, and if the transition between the two options was possible.

The primary source of any motions in the event was a small eruptive filament located deep inside the active region's magnetic core. The transmission of the motions from the filament outward was obviously wavelike, as Figures 8 , and 10 demonstrated. The wave front appeared conspicuously earlier than the rim, not vice versa, as one might expect from the traditional view on the cavity. The rim appears to be formed as the approach of the trajectories of plasma structures 1-4, which started expanding after the passage of the wave front. The earlier a structure started to move, the earlier it disappeared because of a rapid decrease of the emission measure in expanding loops. Eventually, the rim became the outer envelope of the loops, whose behavior was similar.

The wave trajectory in Figure 9 originates at the 'Rope' trajectory corresponding to the top of the eruptive filament. Later it decelerated, but the wave trajectory did not respond to this kinematical change. Thus, the role of the piston was transferred from the eruptive filament to the forming rim. By connecting the trajectories of the observed flux rope and the rim with a single line corresponding to the so-called virtual piston, we get a solution of a known single-piston problem (see, e.g., Sedov, 1981). The trajectories of the wave front and virtual piston intersect, when the piston was the filament-associated rope. This confirms that the primary source of the wave was the observed flux rope developed from the erupting filament inside the cavity, and not the rim, i.e., its outer boundary.

In summary, the role of the major piston responsible for the wave excitation and formation of the rim was played by the small erupting filament, which occupied the central part of the cavity. As time elapsed, this piston dilated, acquiring a clinging magnetic shell, and the whole volume of the cavity became the virtual piston, whose surface became the rim. This scenario seems to present consensus between the two different concepts.

We do not consider the wave excitation scenario by the flare pressure pulse. In a solar flare occurring due to magnetic reconnection, it does not seem possible 
to produce the plasma pressure considerably exceeding the magnetic pressure. For this reason, the increase in the volume of flare loops is insufficient to produce an appreciable MHD disturbance outward (see, e.g., Grechnev et al., 2015, for more detail, see Grechnev et al., 2006b, 2011b, 2014b). The plasma density and temperature in flare loops are manifested in their SXR emission. It is intrinsically gradual, resembling the antiderivative of the HXR burst (the Neupert effect; Neupert, 1968), which roughly corresponds to the acceleration of an eruption responsible for a strong MHD disturbance. As Figures [6 and 6 b show, the flare in this event has not yet developed, when the wave appeared.

\subsection{What Was the Rim?}

Two observational facts indicate a close association between the rim and a separatrix surface bounding the magnetic domain, in which the pre-eruption filament resided. i) There is a visible shear between magnetic structures inside the rim and outside it (Section 3.2). ii) The size of the photospheric base of the cavity did not change in the course of the eruption.

There are additional important indications. iii) Among the loops visible in two dimensions (2D) in the AIA_131_171_loops.mpg movie, their envelope only coincided with the boundary of the cavity. This situation reflects the fact that in the 3D geometry, the outer envelope of the loops belonging to a single domain is its separatrix surface. iv) A turbulence-like wave trail is expected running along the separatrix surface, following the rising spheroidal cavity. The wave trail should appear due to plasma motions in 3D loops belonging to adjacent magnetic domains. These structures should deviate aside and back, as Figure 20 schematically shows. A trail running along the rim is really visible in Figure 18 (circled) and in the running-difference AlA_131_211_dist.mpg movie (the asymmetric arc in the movie connects the top of the eruption with the trails on both sides). The trail running along the south part of the rim is indicated by the arrow, and the north trail resembles turbulence. These features altogether strengthen an impression of an oblate shape of the rim.

Intriguing was the idea of Patsourakos, Vourlidas, and Stenborg (2010) that 'the lateral overexpansion may well be the process through which eruptions starting small in the corona become large-scale CMEs further out'. However, this observed phenomenon does not seem to be a physically significant factor for the CME formation for the following reasons.

i) As shown in Section 5.1, the decrease of the aspect ratio in Figure 10 $\mathrm{d}$, characterizing the apparent ellipticity of the rim, most likely, was a reversible temporary effect. One of its causes could be a dampened twisting rotation and writhe of the erupting filament-related rope inside the cavity. Indeed, its liftoff direction rapidly turned during the temporary decrease in the aspect ratio.

ii) The apparent ellipticity of the cavity in the 13 June 2010 event might be due to its observed geometry with some extent inclined to the line of sight. Note that the cavity in the analog of our event presented by Dere et al. (1997) in their Figure 2 was perfectly spherical.

iii) One might relate the increasing ellipticity of the cavity to the bow-shock regime, when the dynamic pressure on the frontal part of the body is important. 


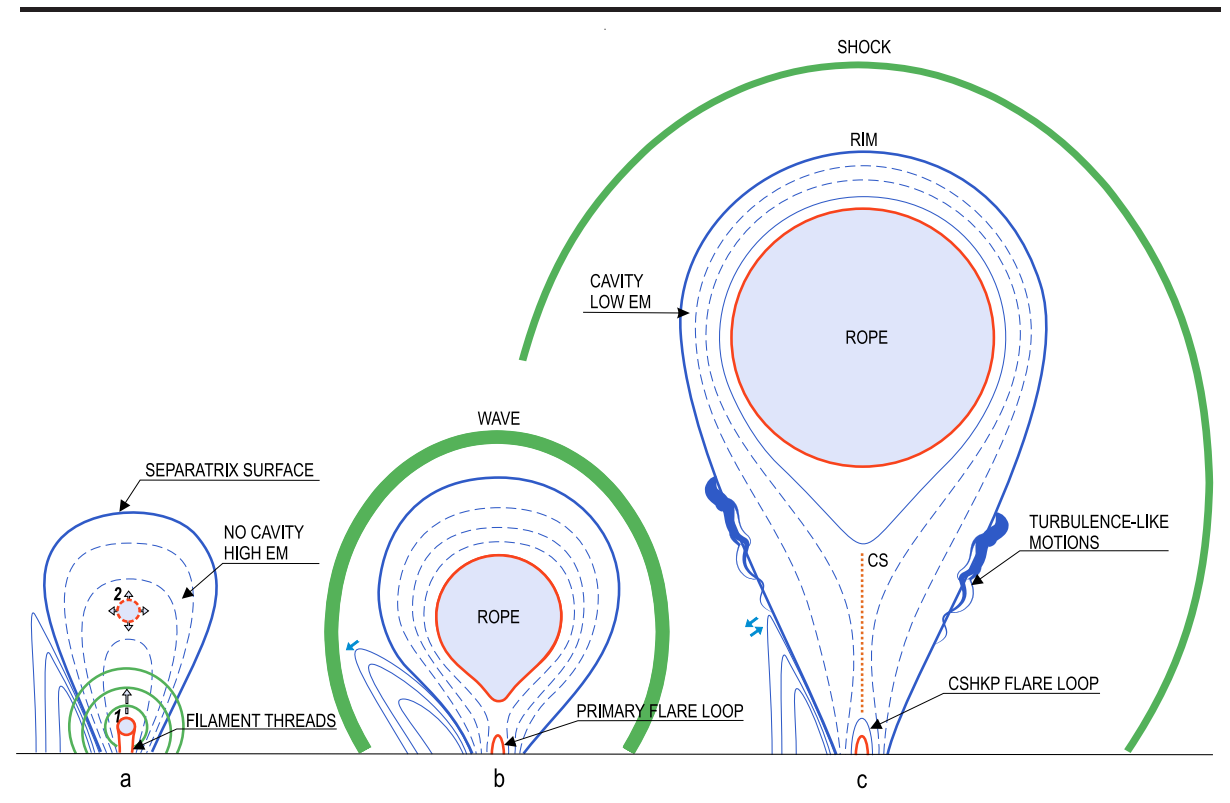

Figure 20. A presumable scenario of the observed event, in which the major driver of the flare, coronal wave, cavity, rim, and CME was a small eruptive filament-associated rope. The cartoon presents a cross section of the active region and the developing CME at three instances. See text for details.

However, the bow-shock regime is ruled out by the dissimilar evolutions of the wave and rim (CME) speeds in Figure $11 \mathrm{~b}$.

iv) As Grechnev et al. (2011a) showed, the shock front can be oblate in the radial direction due to the fast-mode speed distribution. This effect can also contribute to the impression of the lateral overexpansion (see, e.g., Figure 17b).

Thus, a number of different reasons can result in a visual effect of the lateral overexpansion. In any case, the varying shape of the arcade loops and the rim, which were initially passive, presents their response to the disturbances produced by the flux rope inside them. These variations are of a secondary importance, in contrast to the hypothesis relating the outer surface of the active flux rope to the rim.

\subsection{Overall Scheme}

Figure 20 summarizes the results of our analysis of the 13 June 2010 event, starting from the onset of the eruption. The initiation phase with a long-lasting filament heating is not considered. A new key item in this scheme is a dominant, rather than passive, role of a small filament in the formation of the classical structural CME components. These are the coronal cavity, the rim with the frontal structure, and the coronal wave. The filament associated with a core of this configuration determines the subsequent evolution of all these components.

Figure 20 presents a vertical cross-section of the active region's magnetic core. The base corresponds to the photosphere. The thin solid and dashed lines represent the magnetic field lines. The thick blue line is a separatrix bounding 
the major magnetic domain. Figure 20 presents in the lower part of the domain a pre-eruption filament 1 (small red circle), which is a rope-like structure rooted in the photosphere by its lateral threads (red). There is no visible cavity in the initial state; the plasma emission measure in the major domain is rather high. The electric current flowing along the separatrix surface is insignificant. Magnetic loops of an adjacent domain are shown on the left.

Reconnection between the filament threads creates a primary flare loop (red in Figures $20 \mathrm{~b}$ and $20 \mathrm{r}$ ) and primary ribbons, heats the filament, and transforms it into a flux rope (contoured by the red line), which is not connected to the photosphere laterally. Then the flare develops as in the CSHKP model.

Figures 20, and 20b show the expansion and lift-off of the flux rope in two stages. (a) The rope rises, keeping its size and a large internal magnetic pressure. (b) Then the rope expands, equalizing the internal and external pressure. The lift-off process consists of many such steps. The deformations corresponding to each step are transferred outward by fast MHD waves (green ovals in Figure 20 a). Each following wave overtakes a preceding one. The front of a resulting disturbance can become a shock discontinuity (green oval in Figure 20k).

The blue dashed lines between the red circle and thick blue separatrix represent the loops. The expansion and stretch of the loops surrounding the rope is accompanied by their pressing to each other and a decrease in their emission measure. The loops sequentially disappear. The major domain transforms into a cavity bounded by the separatrix surface observed as the rim. Its vicinity is, in fact, a moving current sheet separating magnetic field lines of different connectivity. The current sheet can heat the surrounding plasma. The blue arrows in the left parts of Figures 20b and 20k indicate deviations of the loops during the passage of the spheroidal cavity that results in the propagation of a turbulence-like trail along the separatrix.

This scenario and the cartoon in Figure 20 present the major aspects of the CME and wave development only, and do not account for all phenomena observed in the event. For example, the changing lift-off direction and rotation of the flux rope are not shown. The behavior of the whole system in this case can be understood, keeping in mind that adjacent magnetic surfaces slip along each other.

A subsequent story of CMEs and related waves seems to be more or less clear. Our view, expectations, and results can be found, e.g., in Grechnev et al. (2011b, 2013a, 2014b, 2015). The CME should enter the stage of a free expansion, which is known to be close to self-similar, i.e., the distances between all of its structural components progressively increase. Most likely, the free expansion regime of the 13 June 2010 CME has not yet established within the AIA field of view. Later on, the loops, which disappeared, apparently merging to the rim, should reappear and diverge in the self-similar regime. The expectations are consistent with the LASCO observations of the CME discussed in Section 5.2. For an independent verification of our conclusions related to the waves, see, e.g., the studies by Kwon et al. (2013); Kwon, Zhang, and Olmedo (2014); Kwon, Zhang, and Vourlidas (2015), and others mentioned in the text. 


\subsection{Comparison with Different Observations}

Most of the items of the outlined scenario has been observed in different events. The long-lasting heating of pre-eruptive filaments manifesting in the rise of the SXR flux, firstly stated by Zhang et al. (2001), seems to be rather common. It was shown explicitly or implicitly by, e.g., Kundu et al. (2009), Meshalkina et al. (2009), and Grechnev et al. (2014a, 2015). Erupting filaments often become bright in 171 or $195 \AA$ which indicates their heating up to, at least, 1 MK (see, e.g., the movies at http://trace.Imsal.com/POD/TRACEpod.html\#movielist). Their brightness in $195 \AA$ A well before the flare peak occasionally exceed the flare arcades observed later (Chertok, Belov, and Grechnev, 2015).

A completely formed flux rope identical to the erupted one unlikely preexist because of a large excess of the reconnected over pre-existing poloidal flux (Qiu et al., 2007, Miklenic, Veronig, and Vršnak, 2009) and a strong related propelling Lorentz force. Genesis of eruptive flux ropes from hot parts of filaments or, at least, their association has been reported (Kumar et al., 2012, Cheng et al., 2013, Chen, Bastian, and Gary, 2014). A flux-rope progenitor is not always observed as a filament, probably, due to its higher temperature and lower density, but this should not affect the flux-rope formation.

Erupting filaments and associated hot flux ropes vigorously twist, writhe, and expand sharper and earlier than any surrounding structures. This demonstrates their role as internal drivers of the CME formation process (Cheng et al. 2011, 2013 Zhang, Cheng, and Ding, 2012). The deformations in the magnetic configuration caused by the erupting flux rope are transferred to the environment by a fast-mode MHD wavelike disturbance propagating outward, whose manifestations can be detected far outside. A static coronal structure pushed by the wave unlikely can drive this wave just afterwards. The change to the piston-driven shock is possible later, when the regime of plasma extrusion by the CME bubble changes to the plasma flow around it, if the CME speed exceeds the ambient fast-mode speed (Grechnev et al., 2015).

To verify our results and check if they really help in understanding eruptive phenomena, we briefly consider the SOL2010-11-03 event. Its flare site at S19 E98 was partly occulted. This largely reduced the flare emission with an estimated importance of M5.8 (Chertok, Belov, and Grechnev, 2015) to the actually observed C4.9 level, favoring the observations of an erupting flux rope, but hid its early development. Cheng et al. (2011) revealed a hot flux rope, which rapidly moved outward and compressed the surrounding coronal plasma to form the pileup observed as a bright rim in lower-temperature channels, similar to our event.

Zimovets et al. (2012) measured the average speed of the flux rope within the AIA field of view of $\approx 500 \mathrm{~km} \mathrm{~s}^{-1}$ and the leading edge of the EUV transient of up to $1400 \mathrm{~km} \mathrm{~s}^{-1}$. On the other hand, the white-light CME was as slow as $241 \mathrm{~km} \mathrm{~s}^{-1}$ according to the CME catalog. This fact not considered previously shows that the CME decelerated even more than in our event.

Nevertheless, the event produced a shock wave, which manifested in a bandsplit type II burst. From the position of its source observed in radio images above the CME top, Bain et al. (2012) proposed that the shock with an estimated speed 
of $1900-2000 \mathrm{~km} \mathrm{~s}^{-1}$ was piston-driven (that is difficult to reconcile with a low speed of the white-light CME). Conversely, Zimovets et al. (2012) concluded that the shock wave was initially driven by the eruptive plasmas, but later transformed to a freely propagating blast shock wave, that is very close to the scenario we describe. They also concluded that the band splitting was preferably due to emissions from the regions upstream/downstream of the shock.

Bain et al. (2012) and Zimovets et al. (2012) did not consider a cause of the narrow bandwidth of the type II burst, especially important to reveal the split bands. As shown in Section 6.2 this is only possible if its source is compact, being located, e.g., in a coronal streamer. Unlike our event, where the probable type II source was a remote streamer hit by a quasi-perpendicular shock, the type II emission in the SOL2010-11-03 event seems to have been produced by a quasi-parallel shock in the main streamer above the parent active region. This situation was discussed by Grechnev et al. (2014b; Figure 10); its particularity is a considerably higher drift rate, which was really the case in the SOL2010-11-03 event.

\section{Conclusions}

Taking advantage of the detailed multi-instrument observations of the 13 June 2010 event with an unprecedented temporal and spectral coverage, primarily thanks to the SDO/AIA data, it has become possible to reveal a consistent picture of a solar eruption, coordinating qualitatively and quantitatively its various observational aspects. The inferred scenario updates and specifies existing hypotheses. Unlike traditional expectations, the major driver of the flare, CME formation, and a large-scale wave was the erupting filament. It heated up to $10 \mathrm{MK}$ and even higher; being a direct progenitor of a hypothesized flux rope, the filament transformed into a bundle of erupting loops, which sharply expanded and thus produced a strong MHD disturbance inside the developing CME. This outward-propagating disturbance passed through the forming CME and ran ahead of all its structures. Probably, the disturbance rapidly steepened into a shock resembling a blast wave, produced a type II burst and EUV wave. The magnetic domain containing the eruptive filament/flux rope was forced to expand from inside and became the CME cavity bounded by a separatrix surface observed as a rim. With a nearly spheroidal or pyriform shape, its appearance depends little on the flux-rope orientation. The enhanced-temperature coronal loops above were swept up by the expanding rim and became the CME frontal structure.

Although the envisioned identity of the cavity with a flux-rope has not been confirmed, the role of its rim was important. Being not permeable for plasma, the expanding separatrix surface associated with the rim was responsible for the appearance of dimming in the rarified volume behind it, and took the role of a piston after the deceleration of the flux rope. If the CME were fast, then the wave ahead it eventually changed to the bow-shock regime and became the CME-driven shock in a correct sense. This has not happened, because the CME was slow; the decelerating wave dampened and decayed into a weak disturbance. 
In spite of a similarity between the extremities of the bow-shock and blastwave regimes, some of their properties are different. In particular, when the blast wave is getting weaker, then the distance between its front and the piston decreases. The relation is opposite in the bow-shock regime. For this reason, assumptions of the bow-shock properties for the waves impulsively excited by eruptions might result in an incorrect outcome.

The preceding studies of the 13 June 2010 eruptive event have revealed its important aspects. However, the flux rope, which was a key item of the eruptive process, escaped detection. Due to this difficulty, the researchers had to invoke some traditional assumptions, not all of which have been confirmed. It was also very difficult to detect the flux rope for us. It has become possible, because our persistent search was guided by the expectations based on our preceding results (e.g., Grechnev et al., 2011b, 2014a, 2014b, 2015), and in this way we elaborated the data processing and analysis techniques outlined in Section 2. These techniques might also be helpful in different studies.

Most likely, the updated scenario of an eruptive event presented here is rather typical. Incorporating its items into theoretical considerations and numerical simulations seems to be promising for a better understanding solar eruptive phenomena. Observational and theoretical studies of the causes and mechanisms of the long-lasting pre-eruptive heating, which we did not analyze, could help in perceiving the triggers of solar eruptions and their practical forecasting.

Acknowledgements We thank S.A. Anfinogentov, N.S. Meshalkina, and M.V. Eselevich for their assistance in data handling; and A. Kouloumvakos with co-authors and M.A. Livshits for useful discussions. We are indebted to an anonymous reviewer for the cooperation in bringing this article to its final form. We thank the instrumental teams operating AIA and HMI on SDO, STEREO/SECCHI, Fermi, SOHO/LASCO, Nobeyama Solar Facilities, NICT, USAF RSTN network, and GOES satellites for the data used here. The CME catalog used in this article is generated and maintained at the CDAW Data Center by NASA and The Catholic University of America in cooperation with the Naval Research Laboratory. SOHO is a project of international cooperation between ESA and NASA. This study was supported by the Program of Basic Research No. II.16.1.6, the Integration Project of RAS SD No. 4, and the Russian Foundation of Basic Research under grants 15-02-01077, 15-02-01089, and 15-32-20504-mol-a-ved.

\section{References}

Afanasyev, A.N., Uralov, A.M.: 2012, Solar Phys. 280, 561. DOI: 10.1007/s11207-012-0022-9 Afanasyev, A.N., Uralov, A.M., Grechnev, V.V.: 2013, Astron. Rep., 57, 594. DOI: $10.1134 / \mathrm{S} 1063772913080015$

Alissandrakis, C.E., Kochanov, A.A., Patsourakos, S., Altyntsev, A.T., Lesovoi, S.V., Lesovoya, N.N.: 2013, Publ. Astron. Soc. Japan 65, SP1, S8. DOI: 10.1093/pasj/65.sp1.S8

Anzer, U.: 1978, Solar Phys. 57, 111. DOI: 10.1007/BF00152048

Asai, A., Yokoyama, T., Shimojo, M., Masuda, S., Kurokawa, H., Shibata, K.: 2004, Astrophys. J. 611, 557. DOI: $10.1086 / 422159$

Bain, H.M., Krucker, S., Glesener, L., Lin, R.P.: 2012, Astrophys. J. 750, 44. DOI: 10.1088/0004-637X/750/1/44

Boerner, P., Edwards, C., Lemen, J., Rausch, A., Schrijver, C., Shine, R., Shing, L., Stern, R., Tarbell, T., Title, A. et al.: 2012, Solar Phys. 275, 41. DOI: 10.1007/s11207-011-9804-8

Brueckner, G.E., Howard, R.A., Koomen, M.J., Korendyke, C.M., Michels, D.J., Moses, J.D., Socker, D.G., Dere, K.P., Lamy, P.L., Llebaria, A., et al.: 1995, Solar Phys. 162, 357. DOI: 10.1007/BF00733434

Carmichael, H.: 1964, Proc. of AAS-NASA Symp. on the Physics of Solar Flares, W. N. Hess, NASA-SP 50, 451. 
Chen, J.: 1989, Astrophys. J. 338, 453. DOI: 10.1086/167211

Chen, J.: 1996, J. Geophys. Res. 1012, 27499. DOI: 10.1029/96JA02644

Chen, B., Bastian, T.S., Gary, D.E.: 2014, Astrophys. J. 794, 149. DOI: 10.1088/0004$637 \mathrm{X} / 794 / 2 / 149$

Chen, Y., Du, G., Feng, L., Feng, S., Kong, X., Guo, F., Wang, B., Li, G.: 2014, Astrophys. J. 787, 59. DOI: 10.1088/0004-637X/787/1/59

Cheng, X., Zhang, J., Ding, M.D., Liu, Y., Poomvises, W.: 2013, Astrophys. J. 763, 43. DOI: 10.1088/0004-637X/763/1/43

Cheng, X., Zhang, J., Liu, Y., Ding, M.D.: 2011, Astrophys. J. Lett.732, L25. DOI: $10.1088 / 2041-8205 / 732 / 2 / L 25$

Chertok, I.M., Belov, A.V., Grechnev, V.V.: 2015, Solar Phys. 290, 1947. DOI: 10.1007/s11207-015-0738-4

Dere, K.P., Brueckner, G.E., Howard, R.A., Koomen, M.J., Korendyke, C.M., Kreplin, R.W., Michels, D.J., Moses, J.D., Moulton, N.E., Socker, D.G., et al.: 1997, Solar Phys. 175, 601. DOI: $10.1023 / \mathrm{A}: 1004907307376$

Downs, C., Roussev, I.I., van der Holst, B., Lugaz, N., Sokolov, I.V.: 2012, Astrophys. J. 750, 134. DOI: $10.1088 / 0004-637 \mathrm{X} / 750 / 2 / 134$

Du, G., Chen, Y., Lv, M., Kong, X., Feng, S., Guo, F., Li, G.: 2014, Astrophys. J. Lett. 793 , L39. DOI: 10.1088/2041-8205/793/2/L39

Dulk, G.A., McLean, D.J.: 1978, Solar Phys. 57, 279. DOI: $10.1007 /$ BF00160102

Eselevich, V., Eselevich, M.: 2012, Astrophys. J. 761, 68. DOI: 10.1088/0004-637X/761/1/68

Eselevich, V.G., Eselevich, M.V.: 2013, Astronomy Reports 57, 860. DOI: 10.1134/S1063772913110012

Feng, S.W., Chen, Y., Kong, X.L., Li, G., Song, H.Q., Feng, X.S., Guo, F.: 2013, Astrophys. J. 767, 29. DOI: 10.1088/0004-637X/767/1/29

Gallagher, P.T., Lawrence, G.R., Dennis, B.R.: 2003, Astrophys. J. Lett. 588, L53. DOI: $10.1086 / 375504$

Gary, G.A.: 2001, Solar Phys. 203, 71. DOI: 10.1023/A:1012722021820

Gopalswamy, N., Nitta, N., Akiyama, S., Mäkelä, P., Yashiro, S.: 2012, Astrophys. J. 744, 72. DOI: $10.1088 / 0004-637 \mathrm{X} / 744 / 1 / 72$

Grechnev, V.V., Afanasyev, A.N., Uralov, A.M., Chertok, I.M., Eselevich, M.V., Eselevich, V.G., Rudenko, G.V., Kubo, Y.: 2011a, Solar Phys. 273, 461. DOI: 10.1007/s11207-0119781-y

Grechnev, V.V., Kiselev, V.V., Uralov, A.M., Meshalkina, N.S., Kochanov, A.A.: 2013a, Publ. Astron. Soc. Japan 65, SP1, S9. DOI: 10.1093/pasj/65.sp1.S9

Grechnev, V.V., Uralov, A.M., Chertok, I.M., Kuzmenko, I.V., Afanasyev, A.N., Meshalkina, N.S., Kalashnikov, S.S., Kubo, Y.: 2011b, Solar Phys. 273, 433. DOI: 10.1007/s11207-0119780-z

Grechnev, V.V., Uralov, A.M., Chertok, I.M., Slemzin, V.A., Filippov, B.P., Egorov, Ya.I., Fainshtein, V.G., Afanasyev, A.N., Prestage, N., Temmer, M.: 2014b, Solar Phys. 289, 1279. DOI: $10.1007 / \mathrm{s} 11207-013-0397-2$

Grechnev, V.V., Uralov, A.M., Kuzmenko, I.V., Kochanov, A.A., Chertok, I.M., Kalashnikov, S.S.: 2015, Solar Phys. 290, 129. DOI: 10.1007/s11207-014-0621-8

Grechnev, V.V., Uralov, A.M., Slemzin, V.A., Chertok, I.M., Filippov, B.P., Rudenko, G.V., Temmer, M.: 2014a, Solar Phys. 289, 289. DOI: 10.1007/s11207-013-0316-6

Grechnev, V.V., Uralov, A.M., Slemzin, V.A., Chertok, I.M., Kuzmenko, I.V., Shibasaki, K.: 2008b, Solar Phys. 253, 263. DOI: 10.1007/s11207-008-9178-8

Grechnev, V.V., Uralov, A.M., Zandanov, V.G., Baranov, N.Y., Shibasaki, K.: 2006a, Publ. Astron. Soc. Japan 58, 69. DOI: 10.1093/pasj/58.1.69

Grechnev, V.V., Uralov, A.M., Zandanov, V.G., Rudenko, G.V., Borovik, V.N., Grigorieva, I.Y., Slemzin, V.A., Bogachev, S.A., Kuzin, S.V., Zhitnik, I., Pertsov, A.A., Shibasaki, K., Livshits, M.A.: 2006b, Publ. Astron. Soc. Japan 58, 55. DOI: 10.1093/pasj/58.1.55

Hannah, I.G., Kontar, E.P.: 2012, Astron. Astrophys. 539, A146. DOI: 10.1051/0004$6361 / 201117576$

Hirayama, T.: 1974, Solar Phys. 34, 323. DOI: 10.1007/BF00153671

Howard, R.A., Moses, J.D., Vourlidas, A., Newmark, J.S., Socker, D.G., Plunkett, S.P., Korendyke, C.M., Cook, J.W., Hurley, A., Davila, J.M., et al.: 2008, Space Sci. Rev. 136, 67. DOI: $10.1007 / \mathrm{s} 11214-008-9341-4$

Hudson, H.S., Webb, D.F.: 1997, in: N. Crooker, J. A. Joselyn, and J. Feynman (eds.), Coronal Mass Ejections. Washington DC American Geophysical Union Geophysical Monograph Series 99, 27. DOI: 10.1029/GM099p0027 
Inhester, B., Birn, J., Hesse, M.: 1992, Solar Phys. 138, 257. DOI: 10.1007/BF00151915

Kaiser, M.L., Kucera, T.A., Davila, J.M., St. Cyr, O.C., Guhathakurta, M., Christian, E.: 2008, Space Sci. Rev. 136, 5. DOI: 10.1007/s11214-007-9277-0

Knock, S.A., Cairns, I.H.: 2005, J. Geophys. Res. A 110, A01101. DOI: 10.1029/2004JA010452

Kochanov, A.A., Anfinogentov, S.A., Prosovetsky, D.V., Rudenko, G.V., Grechnev, V.V.: 2013, Publ. Astron. Soc. Japan 65, SP1, S19. DOI: 10.1093/pasj/65.sp1.S19.

Kopp, R.A., Pneuman, G.W.: 1976, Solar Phys. 50, 85. DOI: 10.1007/BF00206193

Kouloumvakos, A., Patsourakos, S., Hillaris, A., Vourlidas, A., Preka-Papadema, P., Moussas, X., Caroubalos, C., Tsitsipis, P., Kontogeorgos, A.: 2014, Solar Phys. 289, 2123. DOI: $10.1007 / \mathrm{s} 11207-013-0460-\mathrm{z}$

Kozarev, K.A., Korreck, K.E., Lobzin, V.V., Weber, M.A., Schwadron, N.A.: 2011, Astrophys. J. Lett. 733, L25. DOI: 10.1088/2041-8205/733/2/L25

Kumar, P., Cho, K.-S., Bong, S.-C., Park, S.-H., Kim, Y.H.: 2012, Astrophys. J. 746, 67. DOI: 10.1088/0004-637X/746/1/67

Kundu, M.R., Grechnev, V.V., White, S.M., Schmahl, E.J., Meshalkina, N.S., Kashapova, L.K.: 2009, Solar Phys. 260, 135. DOI: 10.1007/s11207-009-9437-3

Kwon, R.-Y., Ofman, L., Olmedo, O., Kramar, M., Davila, J. M., Thompson, B.J., Cho, K.-S.: 2013, Astrophys. J., 766, 55. DOI: 10.1088/0004-637X/766/1/55

Kwon, R.-Y., Zhang, J., Olmedo, O.: 2014, Astrophys. J. 794, 148. DOI: 10.1088/0004$637 \mathrm{X} / 794 / 2 / 148$

Kwon, R.-Y., Zhang, J., Vourlidas, A.: 2015, Astrophys. J. Lett. 799, L29. DOI: 10.1088/2041$8205 / 799 / 2 /$ L29

Lemen, J.R., Title, A.M., Akin, D.J., Boerner, P.F., Chou, C., Drake, J.F., Duncan, D.W., Edwards, C.G., Friedlaender, F.M., Heyman, G.F., et al.: 2012, Solar Phys. 275, 17. DOI: 10.1007/s11207-011-9776-8

Levenberg, K.: 1944, Quart. Appl. Math. 2, 164.

Livshits, M.A., Badalyan, O.G.: 2004, Astron. Rep. 48, 1037. DOI: 10.1134/1.1836027

Longcope, D.W., Beveridge, C.: 2007, Astrophys. J. 669, 621. DOI: 10.1086/521521

Low, B.C.: 1982, Astrophys. J. 254, 796. DOI: 10.1086/159790

Ma, S., Raymond, J.C., Golub, L., Lin, J., Chen, H., Grigis, P., Testa, P., Long, D.: 2011, Astrophys. J. 738, 160. DOI: 10.1088/0004-637X/738/2/160

Mancuso, S., Raymond, J.C.: 2004, Astron. Astrophys. 413, 363. DOI: 10.1051/00046361:20031510

Mandrini, C.H., Pohjolainen, S., Dasso, S., Green, L.M., Démoulin, P., van Driel-Gesztelyi, L., Copperwheat, C., Foley, C.: 2005, Astron. Astrophys. 434, 725. DOI: 10.1051/00046361:20041079

Mann, G., Klassen, A., Aurass, H., Classen, H.-T.: 2003, Astron. Astrophys. 400, 329. DOI: 10.1051/0004-6361:20021593

Marquardt, D.W.: 1963, SIAM J. Appl. Math. 11, 431.

Meegan, C., Lichti, G., Bhat, P.N., Bissaldi, E., Briggs, M.S., Connaughton, V., Diehl, R., Fishman, G., Greiner, J., Hoover, A.S., et al.: 2009, Astrophys. J. 702, 791. DOI: 10.1088/0004-637X/702/1/791

Meshalkina, N.S., Uralov, A.M., Grechnev, V.V., Altyntsev, A.T., Kashapova, L.K.: 2009, Publ. Astron. Soc. Japan 61, 791. DOI: 10.1093/pasj/61.4.791

Miklenic, C.H., Veronig, A.M., Vršnak, B.: 2009, Astron. Astrophys. 499, 893. DOI: 10.1051/0004-6361/200810947

Neupert, W.M.: 1968, Astrophys. J. Lett. 153, L59. DOI: 10.1086/180220

Patsourakos, S., Vourlidas, A., Stenborg, G.: 2010, Astrophys. J. Lett. 724, L188. DOI: 10.1088/2041-8205/724/2/L188

Plowman, J., Kankelborg, C., Martens, P.: 2013, Astrophys. J. 771, 2. DOI: 10.1088/0004$637 \mathrm{X} / 771 / 1 / 2$

Qiu, J., Hu, Q., Howard, T.A., Yurchyshyn, V.B.: 2007, Astrophys. J. 659, 758. DOI: $10.1086 / 512060$

Reiner, M.J., Vourlidas, A., St. Cyr, O.C., Burkepile, J.T., Howard, R.A., Kaiser, M.L., Prestage, N.P., Bougeret, J.-L.: 2003, Astrophys. J. 590, 533. DOI: 10.1086/374917

Saito, K.: 1970, Ann. Tokyo Astr. Obs. 12, 53.

Sedov, L.I.: 1981, Similarity Methods and Dimensional Analysis in Mechanics, 9th edn., Nauka, Moscow, 448 pp. (in Russian).

Sheeley, N.R., Jr., Warren, H.P., and Wang, Y.-M.: 2007, Astrophys. J. 671, 926. DOI: $10.1086 / 522940$ 
Smerd, S.F., Sheridan, K.V., Stewart, R.T.: 1974, In: Newkirk, G.A. (ed.) Coronal Disturbances, IAU Symp. 57, Reidel, Dordrecht, 389.

Sterling, A.C., Hudson, H.S.: 1997, Astrophys. J. Lett. 491, L55. DOI: 10.1086/311043

Sturrock, P.A.: 1966, Nature 211, 695. DOI: 10.1038/211695a0

Temmer M., Veronig, A.M., Kontar, E.P., Krucker, S., Vršnak, B.: 2010, Astrophys. J. 712, 1410. DOI: 10.1088/0004-637X/712/2/1410

Temmer, M., Veronig, A.M., Vršnak, Rybák, J., Gömöry, J., Stoiser, S., Maričić, D.: 2008, Astrophys. J. Lett. 673, L95. DOI: 10.1086/527414

Torii, C., Tsukiji, Y., Kobayashi, S., Yoshimi, N., Tanaka, H., Enome, S.: 1979, Proc. Res. Inst. Atmospherics, 26, 129.

Uchida, Y.: 1968, Solar Phys. 4, 30. DOI: 10.1007/BF00146996

Uralov, A.M.: 1990, Solar Phys. 127, 253. DOI: 10.1007/BF00152165

Uralov, A.M., Grechnev, V.V., Hudson, H.S.: 2005, J. Geophys. Res. 110, A05104. DOI: 10.1029/2004JA010951

Uralov, A.M., Grechnev, V.V., Rudenko, G.V., Myshyakov, I.I., Chertok, I.M., Filippov, B.P., Slemzin, V.A.: 2014, Solar Phys. 289, 3747. DOI: 10.1007/s11207-014-0536-4.

Uralov, A.M., Lesovoi, S.V., Zandanov, V.G., Grechnev, V.V.: 2002, Solar Phys. 208, 69. DOI: 10.1023/A:1019610614255

Uralova, S.V., Uralov, A.M.: 1994, Solar Phys. 152, 457. DOI: 10.1007/BF00680450

van Ballegooijen, A.A., Martens, P.C.H.: 1989, Astrophys. J. 343, 971. DOI: 10.1086/167766

Vasanth, V., Umapathy, S., Vršnak, B., Žic, T., Prakash, O.: 2014, Solar Phys. 289, 251. DOI: $10.1007 / \mathrm{s} 11207-013-0318-4$

Vršnak, B., Cliver, E.W.: 2008, Solar Phys. 253, 215. DOI: 10.1007/s11207-008-9241-5

Vršnak, B., Maričić, D., Stanger, A.L., Veronig, A.M., Temmer, M., Roša, D.: 2007, Solar Phys. 241, 85. DOI: 10.1007/s11207-006-0290-3

Warmuth, A.: 2015, Living Reviews in Solar Physics 12, 3. DOI: 10.1007/lrsp-2015-3

Warmuth, A., Vršnak, B., Aurass, H., Hanslmeier, A.: 2001, Astrophys. J. Lett. 560, L105. DOI: $10.1086 / 324055$

Wang, Y., Zhang, J., Shen, C.: 2009, J. Geophys. Res. 114, 10104. DOI: 10.1029/2009JA014360

Webb, D.F., Lepping, R.P., Burlaga, L.F., DeForest, C.E., Larson, D.E., Martin, S.F., Plunkett, S.P., Rust, D.M.: 2000, J. Geophys. Res. 105, 27251. DOI: 10.1029/2000JA000021

Yashiro, S., Gopalswamy, N., Michalek, G., St. Cyr, O.C., Plunkett, S.P., Rich, N.B., Howard, R.A.: 2004, J. Geophys. Res. 109, A07105. DOI: 10.1029/2003JA010282

Zimovets, I., Vilmer, N., Chian, A.C.-L., Sharykin, I., Struminsky, A.: 2012, Astron. Astrophys. 547, A6. DOI: 10.1051/0004-6361/201219454

Zhang, J., Cheng, X., Ding, M.-D.: 2012, Nat. Comm. 3, 747. DOI: 10.1038/ncomms1753

Zhang, J., Dere, K.P., Howard, R.A., Kundu, M.R., White, S.M.: 2001, Astrophys. J. 559, 452. DOI: $10.1086 / 322405$ 
SOLA: 2010-06-13_prep.tex; 15 March 2018; 2:25; p. 46 